\title{
THE QUANTITATIVE ANALYTICS OF THE BASIC NEOMONETARIST MODEL
}

\author{
Miles S. Kimball
}

Working Paper No. 5046

\section{NATIONAL BUREAU OF ECONOMIC RESEARCH 1050 Massachusetts Avenue \\ Cambridge, MA 02138 \\ February 1995}

This paper is part of NBER's research programs in Economic Fluctuations and Monetary Economics. Any opinions expressed are those of the author and not those of the National Bureau of Economic Research.

() 1995 by Miles S. Kimball. All rights reserved. Short sections of text, not to exceed two paragraphs, may be quoted without explicit permission provided that full credit, including $\odot$ notice, is given to the source. 
NBER Working Paper \#5046

February 1995

\title{
THE QUANTITATIVE ANALYTICS OF THE BASIC NEOMONETARIST MODEL
}

\begin{abstract}
This paper constructs a dynamic macroeconomic model with less-than-perfect price flexibility which has a real side consistent with Real Business Cycle Theory, augmented by investment adjustment costs, increasing returns to scale, and a new, flexible formalization of imperfect competition. A new mode of approximation is developed-useful for any model in which one state variable adjusts quickly, while another state variable adjusts slowly. Even with investment adjustment costs, monetary expansions are found to raise the real interest rate. The determinants of real rigidity and the macroeconomic rate of price adjustment are investigated.
\end{abstract}

Miles S. Kimball

Department of Economics

University of Michigan

Ann Arbor, MI 48109-1220

and NBER 


\title{
The Quantitative Analytics of the Basic Neomonetarist Model
}

\author{
Miles Kimball \\ University of Michigan
}

January 1995

A great deal of macroeconomic research in the last decade or $t$ wo has been motivated by a lively debate over whether or not prices are sticky and whether and how much money matters. What are the lessons those who have not been active partisans can take away from this debate? I cannot claim true impartiality, because of my saltwater graduate school background on one side and my attraction to the elegance of the real business cycle picture of an economy of optimizing agents responding to the fundamental economic forces of preferences and technology on the other. But like a bystander to the battle who moves among the dead and wounded to see what valuables he can gather, I have tried to collect things of worth from both sides. Thus, this paper is meant to stimulate discussion, not to be the final word on anything.

I will argue that a hybrid model should be taken much more seriously-a model following the Real Business Cycle paradigm as closely as possible except for adding what is logically necessary in order to graft in sticky prices. In order to avoid implicating either the New Keynesians or the New Classicals in my crimes, I will call such a hybrid model-grafting sticky prices into what would otherwise be a Real Business Cycle model-a "Neomonetarist" model. Such models are not unknown in the literature, but they have not been studied by as many people with as great intensity as other models. To justify more attention to such hybrid models, I must defend the assumption of sticky prices and defend the essence of the Real Business Cycle approach.

The main reason to be interested in sticky prices is that they provide one of the few means of generating large monetary nonneutralities. The basic evidence for the existence of large monetary nonneutralities is the evidence assembled by Friedman and Schwartz (1963), together with events after the end of their sample, such as the Volcker deflation. The details of the historical record discussed by Friedman and Schwartz (1963) have persuaded most macroeconomists that there is has been a substantial exogenous component to the timing of monetary policy and sometimes even the direction of monetary policy based on the personalities and the inner workings of the Federal Open Market Committee 
and the Federal Reserve System more generally. The most exogenous shifts in monetary policy seem to have the clearest effects. Such historical evidence for the potency of monetary policy is also available for a host of other countries as well.

Despite considerable effort, there has been little success at generating large monetary nonneutralities in flexible-price models using plausible parameters for anything short of a hyperinflationary situation. The basic problem is that, if prices are perfectly flexible, the monetary services subsector (which is a small part of the financial services sector) looks a lot like any other type of service subsector in the economy. Short of massive bank failures or hyperinflation, the fraction of a percent share monetary services have in $\operatorname{GDP}^{1}$ (a fraction of the share of financial services in general) is simply too small for what happens to this sector to be a big deal if prices are perfectly flexible. (During hyperinflations, monetary services as a share of GDP do become substantial, sometimes rising above $10 \%$ of GDP.) By contrast, if prices are sticky, what happens in the monetary services sector can distort the decisions of firms in all sectors of the economy that have sticky prices. ${ }^{2}$

Real Business Cycle research has sometimes (but not always) been pursued in opposition to the idea of sticky prices, but this approach has many important strengths even if one is willing to entertain the idea that prices are sticky. Real Business Cycle research has consistently taken dynamics, sensible anticipation of the future, quantitative calibration and the welfare implications of models very seriously. As a result, even simple Real Business Cycle models are rich in quantitative dynamic implications. The agreement on the Basic Real Business Cycle model of Prescott (1986) as a benchmark allows researchers to compare their results and communicate in a productive way as they modify one or two features of the basic model. Explicit, disciplined modeling opens the possibility of unexpected results.

Real Business Cycle models may be oversimplified, but it is hard to dismiss the forces identified in such models as important contributors to what is going on. Firms may not always minimize costs, but a tendency toward cost minimization governs much of what happens in the economy. All households may not be rationally forward looking, but an important fraction of households surely do condition their decisions in important ways on expectations about the future. Households may not be on their labor supply curves at every instant, but they surely resist fluctuating too far off their labor supply curves for too

\footnotetext{
1The amount paid for monetary services in proportion to GDP is equal to the rate of foregone intereat times $\frac{M}{P Y}=\frac{1}{V}$. An $M 1$ velocity of 6 , coupled with an average foregone interest for the components of M1 of $5 \%$ would lead to a share of less than $1 \%$ ( $\frac{5}{8} \%$ in this case) for monetary services in GDP.

${ }^{2}$ The literature does give some examples of theoretical models that have large monetary nonneutralities with perfectly flexible prices and no information problems, but these are models with multiple equilibria or very fragile equilibria in which small costs of price adjustment would have an even easier time generating large monetary nonneutralities.
} 
long. Thus, it seems folly to ignore the economic forces highlighted in Real Business Cycle models even if one wishes to add other mechanisms to a model as well. It is particularly difficult to think about investment in a sensible way without borrowing some of the rational expectations apparatus.

In addition to presenting a hybrid model, I will try to hint at a joint research program for New Keynesians and Real Business Cyclists. In both approaches, the key to explaining economic fluctuations is constructing a model with plausible parameters that allows for a strong response to shocks. In both approaches the key model elements generating responsiveness of the economy to shocks are things like favorably shaped firm demand and cost curves, positive externalities, and a fairly large macroeconomic elasticity of labor supply. The challenge of adequately justifying such model elements provides plenty of scope for a joint research agenda.

\section{The Monetary (and Price-Adjustment) Side of the Neomonetarist Model}

In this model I will use two kinds of approximations in order to focus on the most important effects and to have model that is easy to understand. First, I will make a certainty-equivalence approximation throughout, and focus on a linearizing or log-linearizing around steady-state values. ${ }^{3}$ (I will use a tilde () to denote the deviation of a variable from its steady-state value and a haček () to denote the logarithmic deviation of a variable from its steady-state value.) Second, I will look at a Taylor expansion based on the typical length of time a price is fixed being relatively short. ${ }^{4}$ This greatly simplifies many expressions in a very intuitive way. In fact, this high price flexibility approximation is a formal way to justify something akin to the Classical Dichotomy separating real growth and fluctuations on the one hand from fluctuations induced by monetary factors on the other. I will call the aspects of the model that determine its short-run fluctuations in response to monetary factors the monetary side of the Neomonetarist model.

\footnotetext{
${ }^{3}$ Although this is adequate for a first crack at studying the responses of variables to shocks in the Neomonetarist model, it precludes a serious welfare analysis of changes that affect the variance of output, since the fact that under imperfect competition more output is typically beneficial socially means that the influence of depart ures from certainty equivalence on mean output matters for such a welfare analysis. For instance, it is not inconceivable that more variable output could induce enough extra saving through precautionary effects that the larger mean capital stock and higher mean output could more than compensate for the direct cost of higher variance on welfare.

"One advantage of working in continuous time is that it highlights the question of how long a price is likely to be fixed. In discrete time models it is all too tempting to dodge this issue by assuming that prices are set for "one period" without any serious thought given to whether "one period" is in fact the likely length of time for which a price is fixed.
} 


\subsection{Money Demand and Supply}

With velocity $V$ assumed exogenous in the monetarist spirit, the identity

$$
M V=P Y
$$

gets some real bite. $M$, of course, is the money supply, $Y$ is aggregate output and $P$ is the aggregate price level. This equation determines aggregate output as

$$
Y=\frac{M V}{P},
$$

or in logarithmic deviations,

$$
\check{Y}=\check{M}+\check{V}-\check{P} .
$$

As noted above, I assume that the share of money services in GDP is negligible, so that the money market only really matters because of its interaction with sticky prices. This is a reasonable assumption in non-hyperinflationary situations.

At this point, we already have an aggregate demand curve, (1), and the short-run aggregate supply curve is a horizontal at the price sticky price $P$. All of the interest is in the action going on behind the scenes of the aggregate demand and short-run aggregate supply curve.

\subsection{Price Adjustment Dynamics}

I will use Calvo's (1982) model of price adjustment in continuous time. The biggest single reason for doing so is that here, in a model that has other important complicating features, it seems wise to focus on time-dependent setting of fixed prices for the sake of tractability. Looking at the effects of different price-adjustment mechanisms is important, but because of its simplicity, the Calvo model is the place to start.

Let me at least explain why I emphasize sticky prices rather than sticky wages. The less important reason is that wage stickiness tends to yield countercyclical real wages which are not apparent in the data. A deeper and much more important reason for the focus on price stickiness as opposed to wage stickiness is the view I share with many other macroeconomists (both New Keynesian and New Classical) that most employment is part of a long-term implicit contract in which the wage observed at any given time is only an installment payment on what the implicit contract says is due to a worker. Therefore, the observed wage does not accurately represent a firm's labor costs. True marginal labor costs are a matter of the additional amount a firm is implicitly promising to pay a worker someday in return for working an additional hour. Given long-term implicit contracts, sticky-looking wages are a convenience for both workers and firms 
and can even have an insurance role to play, but they have little bearing on true labor costs. Traditional models of sticky wages require not just sticky observed wages, which are easy to justify, but sticky labor costs, which are very difficult to justify.

\subsubsection{The Evolution of the Aggregate Price Level}

In Calvo's (1982) model, each firm gets the chance to consider and adjust its price at an interval determined by a Poisson process, with the chance to adjust the firm's price arriving with probability $\alpha$ per unit time. Because all firms are identical except for the fixed prices they have at the moment, all firms that get the chance to change their price at a given time will choose the same new price, which I will call the optimal reset price and denote $\mathcal{P}_{t}$.

Firms that have fixed prices and can only change them at random intervals will unavoidably end up with different prices whenever there are any fluctuations. The appropriate aggregate for these different prices can be quite complex. Because of the underlying symmetry between the various firms, even a complex price aggregate is equal, to a first-order approximation, to a simple, unweighted arithmetic average of all the prices. The interaction of a firm's change in its price and any departure of the quantity weight for that price from symmetry with the quantity weights for all of the other firms' prices is a second-order effect. ${ }^{5}$ Thus,

$$
\dot{\tilde{P}}_{t}=\alpha\left[\tilde{\mathcal{P}}_{t}-\tilde{P}_{t}\right]
$$

since price changes take place at the Poisson rate $\alpha$ from various prices that average $P_{t}$ to the optimal reset price $\mathcal{P}_{t}$.

\subsubsection{The Optimal Reset Price}

Given the chance, a firm chooses its reset price $\mathcal{P}$ at time $t$ by maximizing the expected present value of profits:

$$
\max _{\mathcal{P}_{1}} \mathrm{E}_{t} \int_{t}^{\infty} e^{-\int_{t}^{p^{\prime}}\left(r_{\bullet}+\alpha\right) d \sigma} \Pi\left(\frac{\mathcal{P}_{t}}{P_{s}}, s\right) d s,
$$

where $E_{t}$ is an expectation over all the events other than the arrival of the chance to reset one's price, conditional on information at time $t, r_{\sigma}$ is the real interest rate at time $s, \Pi$ is the flow of real profits as a function of the firm's price relative to the aggregate price at time $s, P_{s}$. The dependence of real profits on everything else is represented by the second, time argument in $\Pi$. The profits are discounted by $\alpha$ as well as $r$ because the current reset price will last for a length of time $s-t$ only with probability $e^{-\alpha(s-t)}$.

\footnotetext{
${ }^{5}$ Trend inflation, which I have now assumed away, could result in interactions bet ween price changes and asymmetric quantity weights which are technically first-order, but these effects would vanish as $\alpha \rightarrow \infty$.
} 
Using a prime for a derivative of $\Pi$ with respect to its first, relative price argument, the first-order condition for the optimal reset price is

$$
0=\mathrm{E}_{t} \int_{t}^{\infty} e^{-\int_{t}^{\prime}\left(r_{\sigma}+\alpha\right) d \sigma} \frac{\Pi^{\prime}\left(\frac{\mathcal{P}_{t}}{P_{t}}, s\right)}{P_{s}} d s .
$$

The certainty-equivalence approximation makes the impulse-response functions the same as in a perfect-foresight model, so I will drop the expectation sign $E_{t}$ for now. Linearizing marginal profits around a relative price of 1 ,

$$
\Pi^{\prime}\left(\frac{\mathcal{P}_{t}}{P_{s}}, s\right) \approx \Pi^{\prime}(1, s)+\Pi^{\prime \prime}(1, s)\left[\frac{\mathcal{P}_{t}}{P_{s}}-1\right] .
$$

It is helpful to define a "target price" $P_{s}^{\#}$ which would be optimal at a particular instant if the firm could choose a price for just that instant without being stuck with that price at other instants. This instantaneously optimal target price $P_{s}^{\#}$ satisfies the instantaneous first order condition (and its linearizing)

$$
0=\Pi^{\prime}\left(\frac{P_{s}^{\#}}{P_{s}}, s\right) \approx \Pi^{\prime}(1, s)+\Pi^{\prime \prime}(1, s)\left[\frac{P_{s}^{\#}}{P_{s}}-1\right]
$$

Subtracting (5) from (4) yields the approximation

$$
\Pi^{\prime}\left(\frac{\mathcal{P}_{t}}{P_{s}}, s\right) \approx \Pi^{\prime \prime}(1, s)\left(\frac{\mathcal{P}_{t}-P_{s}^{\#}}{P_{s}}\right)
$$

Substituting (6) into the first-order condition (3) with the expectation sign omitted, and using $\pi_{\sigma}$ to denote the rate of inflation in the aggregate price level at time $s$,

$$
\begin{aligned}
0 & =\int_{t}^{\infty} e^{-\int_{t}^{\prime}\left(r_{\sigma}+\alpha\right) d \sigma} \frac{\Pi^{\prime \prime}(1, s)}{P_{s}^{2}}\left[\mathcal{P}_{t}-P_{s}^{\#}\right] d s \\
& =\frac{1}{P_{t}^{2}} \int_{t}^{\infty} e^{-\int_{t}^{\prime}\left(\alpha+r_{\sigma}+2 \pi_{\sigma}\right) d \sigma} \Pi^{\prime \prime}(1, s)\left[\mathcal{P}_{t}-P_{s}^{\#}\right] d s .
\end{aligned}
$$

Solving for the optimal reset price $\mathcal{P}_{t}$,

$$
\mathcal{P}_{t}=\frac{\int_{t}^{\infty} e^{-\int_{t}^{\prime}\left(r_{\sigma}+\alpha\right) d \sigma} \frac{\Pi^{\prime \prime}(1, s)}{P_{t}^{2}} P_{s}^{\#} d s}{\int_{t}^{\infty} e^{-\int_{t}^{\prime}\left(r_{\sigma}+\alpha\right) d \sigma} \frac{\Pi^{\prime \prime}(1, s)}{P_{0}^{2}} d s}
$$

In words, the optimal reset price is (to a linear approximation) a weighted average of the instantaneously optimal target price over the length of time until the next price decision, appropriately discounted. Other than the discounting, the weight is the curvature of the profit function with respect to the relative price, divided by the square of the aggregate price level. The aggregate price 
level is part of the weight because the higher the aggregate price level, the less effect the firm's own price will have on its relative price level.

Alternatively, one can use Leibniz' rule to differentiate (7) with respect to time and then solve for $\dot{\mathcal{P}}_{t}$ :

$$
\dot{\mathcal{P}}_{t}=\frac{1}{\int_{t}^{\infty} e^{-\int_{t}^{\prime}\left(\alpha+r_{\sigma}+2 \pi_{\sigma}\right) d s} \frac{\Pi^{\prime \prime \prime}(1, s)}{\Pi^{\prime \prime}(1, t)} d s}\left[\mathcal{P}_{t}-P_{t}^{\#}\right]
$$

I will use asterisks to denote values in a steady state with constant aggregate real values and a constant rate of inflation $\pi^{*}$. The model is applicable to an economy growing in real terms, but all real quantities must be detrended before being inserted in the model, including the adjustments to the depreciation rate, real interest rate and utility discount rate necessary for a static economy to represent one growing at a steady rate in real terms. (See the chapter in Kimball and Weil (forthcoming) on "Steady States and Growth Steady States.")

Tildes denote deviations from those steady-state values. In finding a firstorder approximation to the fluctuations around this steady state, these deviations can be treated as differentials from calculus. ${ }^{6}$ Equation (9) implies

$$
\begin{aligned}
\frac{\dot{\tilde{P}}_{t}}{\mathcal{P}_{t}^{*}}=\left(\alpha+r^{*}\right. & \left.+2 \pi^{*}\right)\left(\frac{\tilde{\mathcal{P}}_{t}-\tilde{P}_{t}^{\#}}{\mathcal{P}_{t}^{*}}\right) \\
& +\left[1-\left(\frac{P_{t}^{\#}}{\mathcal{P}_{t}}\right)^{*}\right]\left(\frac{1}{\int_{t}^{\infty} e^{-\int_{t}^{*}\left(\alpha+r_{\sigma}+2 \pi_{\sigma}\right) d \sigma} \frac{\Pi^{\prime \prime \prime}(1, s)}{\Pi^{\prime \prime}(1, t)} d s}\right) .
\end{aligned}
$$

If the initial steady state has stable prices $\left(\pi^{*}=0\right)$, the second line of $(10)$ becomes zero, because $\frac{P_{f}^{*}}{\mathcal{P}_{t}}=1$. For the remainder of this paper, I will pursue this linear approximation around a stable price steady-state. For modest rates of trend inflation, the appropriate correction should be small.

\subsection{Market Structure and the Instantaneously Optimal Target Price}

In addition to depending on the level of output in relation to "full-employment" output, the instantaneously optimal target price $P \#$ depends on the model's market structure, broadly construed.

Models with sticky prices also tend to have imperfect competition and increasing returns for good reason. Sticky prices require imperfect competition in order for firms to be price-setters. (Under perfect competition, each firm is a price-taker and the only price-setter is the Walrasian auctioneer.)

${ }^{6}$ The time derivative of such a deviation is equal to the deviation of the time derivative; just as in taking a mixed partial derivative the order of the two operations does not matter. 
Imperfect competition, in turn, is intimately connected by economic logic with internal increasing returns to scale. Internal increasing returns to scale makes firms likely to become large enough to make perfect competition doubtful. Conversely, unless entry is artificially restricted, constant returns to scale would allow firms to enter imperfectly competitive markets (which are attractive because price is above cost) until those markets approach being perfectly competitive.

Besides making sticky prices possible, imperfect competition has a number of other important and attractive implications for business cycle models. First, for some, the fact that imperfect competition makes increasing returns to scale possible is a virtue.

Second, imperfect competition captures the intuition that, over some very relevant range, more output is better. As Weitzman (1984) notes, the eagerness of firms to sell extra units of their products (as evidenced by the considerable efforts devoted to marketing) is prima facie evidence of price being above marginal cost and therefore of imperfect competition in a wide range of industries. The eagerness of firms to provide more at the given price avoids, for the most part, rationing of supply by firms. The eagerness of firms to provide more at the given price, at which households or other firms are eager to buy indicates that output is likely to be socially too low.

Third, the existence of a markup of price over marginal cost raises the possibility that the markup might vary over the business cycle.

A countercyclical actual markup is a likely consequence of sticky prices if marginal cost is procyclical and prices, because of their stickiness are fairly acyclical. The importance of even these undesired fluctuations in the actual markup cannot be overemphasized, because the only important effects of nominal things on the real equations of a Neomonetarist model operate through the fluctuations in the actual markup. In other words, to a first approximation, knowing the actual markup alone tells one enough to solve the model in real terms without knowing anything else from the monetary and nominal side of the model.

In large part because of the things discussed above, macroeconomists have been quite interested in the effects of market structure on economic fluctuations"market structure" including both the shape of an individual firm's demand curve and how the shape of the firm's demand curve changes in response to aggregate forces. Specific macroeconomic investigations of the effects of market structure abound in the literature. Rather than try to deal directly with the great variety of models in the literature, I will use a model of market structure for which the main virtues are simplicity and flexibility in a few important dimensions. Rather than making any particular claim to realism for this model of market structure in and of itself, I hope only that it can represent reasonably well the kind of effects one would see in a more realistic, but also more cumbersome model.

First, for simplicity, I will assume no entry and exit of the differentiated firms 
producing varied products. Second, I will assume that there is a competitive industry that assembles final goods from the many varieties of intermediate good obtained from the differentiated firms. Equivalently, each end user can do the assembly of the final good. No labor or capital are needed to make the final good from the intermediate goods. This becomes less implausible when one realizes that some of the intermediate "goods" may in fact be services.

The quantity $Y$ of the final good produced is given implicitly by

$$
1=\int_{0}^{1} \Upsilon\left(y_{\ell} / Y\right) d \ell
$$

where the differentiated firms and the type of intermediate good coming from each are indexed by $\ell$ on the continuum from 0 to 1 , and the function $\Upsilon$ satisfies $\Upsilon(1)=1, \Upsilon^{\prime}(\xi)>0$ and $\Upsilon^{\prime \prime}(\xi)<0$, for all $\xi \geq 0$. By construction, (11) implies that the final goods production function is constantreturnstoscale in all of the intermediate goods, as befits a competitive industry.

The demand curve each (intermediate good) firm faces can be derived by looking at the optimal choice of intermediate good varieties in the production of final goods. The assembler of the final good minimizes the cost of the intermediate goods necessary to produce any given amount:

$$
\min _{y} \int_{0}^{1} p_{\ell} y_{\ell} d \ell
$$

s.t.

$$
1=\int_{0}^{1} \Upsilon\left(y_{\ell} / Y\right) d \ell
$$

for given $Y$. The first-order condition for this problem is

$$
p_{\ell}=\frac{\Lambda}{Y} \Upsilon^{\prime}\left(y_{\ell} / Y\right)
$$

for some value of the Lagrange multiplier $\Lambda$ that is constant for all $\ell$.

What I need to know about the demand curve facing an individual firm is the behavior of the elasticity of demand. Writing $\xi=y_{\ell} / Y$, the inverse elasticity of demand for a differentiated firm is

$$
\begin{aligned}
\frac{1}{\epsilon(\xi)} & =-y \frac{d \ln \left(p_{\ell}\right)}{d y} \\
& =\frac{y}{Y} \frac{\Upsilon^{\prime \prime}\left(y_{\ell} / Y\right)}{\Upsilon^{\prime}(\nvdash)} \\
& =-\frac{\xi \Upsilon^{\prime \prime}(\xi)}{\Upsilon^{\prime}(\xi)} .
\end{aligned}
$$


(The elasticity calculation depends on the firm being an infinitesimal part of the economy, so that its change in its own price has a negligible effect on $Y$ and

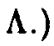

It is easy to find a variety aggregator $\boldsymbol{\Upsilon}$ to match any desired dependence of the elasticity of demand $\epsilon$ on the firm's relative output $\xi$. Viewed as a differential equation for the function $\Upsilon^{\prime}(\xi)$, (13) has the particular solution

$$
\Upsilon^{\prime}(\xi)=\exp \left(-\int_{1}^{\xi} \frac{1}{\zeta \epsilon(\zeta)} d \zeta\right)
$$

In conjunction with the initial condition $\Upsilon(1)=1$, this determines a shape for the function $\Upsilon(\xi)$ that generates any given elasticity function $\epsilon(\xi)$.

The elasticity of demand matters for two reasons. First, the target markup $\mu(\xi)$ that the firm would impose in choosing its instantaneously optimal target price is given by

$$
\mu(\xi)=\frac{1}{1-(1 / \epsilon(\xi))}=\frac{1}{1-\left(\xi \Upsilon^{\prime \prime}(\xi) / \Upsilon^{\prime}(\xi)\right)} .
$$

Second, this elasticity relates movements in relative output $\xi_{\ell}$ to movements in the relative price $p_{\ell} / P$. Log-linearizing (12) around $\xi=1$, with hačeks (, read "check") representing logarithmic deviations, yields

$$
\check{p}_{\ell}=\check{\Lambda}-\check{Y}-\frac{1}{\epsilon(1)} \check{\xi}_{\ell} ;
$$

the price given by the demand-curve falls with the firms relative output. Since the share weights on the prices of each firm are all equal at the steady state,

$$
\begin{aligned}
\check{P} & =\int_{0}^{1} \check{p}_{\ell} \\
& =\int_{0}^{1}\left[\check{\Lambda}-\check{Y}-\frac{1}{\epsilon(1)} \check{\xi}_{\ell}\right] d \ell \\
& =\check{\Lambda}-\check{Y}-\frac{1}{\epsilon(1)} \int_{0}^{1} \check{\xi}_{\ell} \cdot d \ell
\end{aligned}
$$

But, log-linearizing the identity (11) around $\xi=1$ reveals that

$$
\frac{\Upsilon^{\prime}(1)}{\Upsilon(1)} \int_{0}^{1} \dot{\xi}_{\ell} d \ell=0,
$$

or

$$
\int_{0}^{1} \bar{\xi}_{l} d \ell=0
$$


Therefore,

$$
\check{P}=\check{\Lambda}-\check{Y}
$$

and, writing $\epsilon(1)=\epsilon^{*}$,

$$
\begin{aligned}
\check{p}_{\ell}-\check{P} & =\check{\Lambda}-\check{Y}-\frac{\check{\xi}_{\ell}}{\epsilon^{*}} \bar{\xi}^{\#}-[\check{\Lambda}-\check{Y}] \\
& =-\frac{\check{\xi}_{\ell}}{\epsilon^{*}}
\end{aligned}
$$

\subsection{Full Employment Output and Target Output}

At this stage, it is helpful to represent a firm's real marginal cost as a function of a firm's own output relative to aggregate output, aggregate output itself (which can affect marginal cost through factor prices and possibly through production externalities), and everything else. That is,

$$
\frac{\mathrm{MC}}{P}=\Phi(\xi, Y, \cdot)
$$

where $\frac{\xi=y_{e}}{Y}$ as above. As will be justified by later calculations, I will assume that $\Phi_{Y}>0$ : that is, marginal costs are increasing when aggregate output and firm output increase by the same percentage (leaving $\xi$ fixed). If $\Phi_{Y}<0$, it becomes easy to generate multiple equilibria. Even low positive values of the elasticity $\frac{\gamma \Phi_{Y}}{\Phi}$ (which are far from guaranteed) imply interesting macroeconomic behavior, as becomes evident below.

The instantaneously optimal target price $P_{\#}^{\#}$, and the target output ratio $\xi^{\#}=y^{\#} / Y$ that goes along with it, must satisfy the condition that marginal revenue equals marginal cost—or equivalently, that the target mark-up ratio times marginal cost equals the target price:

$$
P^{\#}=\mu\left(\xi^{\#}\right) \Phi\left(\xi^{\#}, Y, \cdot\right) P \text {. }
$$

Let me define "full employment output" $Y^{\prime}$ to be the level of aggregate output at which for given values of all the arguments of $\Phi$ summarized by - the instantaneously optimal target price is equal to the aggregate price level $\left(P^{\#} / P=1\right)$, and the associated target output ratio $\xi^{\#}=y^{\#} / Y$ is 1 . That is, $Y^{f}$ is defined by the equation

$$
1=\mu(1) \Phi\left(1, Y^{f}, \cdot\right)
$$

Dividing (17) by (18),

$$
\ln \left(P^{\#}\right)-\ln (P)=\ln \left(\mu\left(\xi^{\#}\right)\right)-\ln (\mu(1)) \ln \left(\Phi\left(\xi^{\#}, Y, \cdot\right)\right)-\Phi\left(1, Y^{J}, \cdot\right) .
$$


Thus, log-linearizing (17) divided (18) around the (stable-price) steady-state values $\frac{P^{*}}{P}=1, \xi^{\#}=1$ and $Y=Y^{*}$, and $\cdot=\cdot^{*}$ yields

$$
\check{P}^{\#}-\check{P}=\left[\frac{\mu^{\prime}(1)}{\mu(1)}+\frac{\Phi_{\xi}\left(1, Y^{*}, \cdot^{*}\right)}{\Phi\left(1, Y^{*}, \cdot^{*}\right)}\right] \check{\xi}^{\#}+\frac{Y^{*} \Phi Y\left(1, Y^{*}, \cdot^{*}\right)}{\Phi\left(1, Y^{*}, \cdot^{*}\right)}\left[\check{Y}-\check{Y}^{\prime}\right]
$$

By (16)

$$
\check{P}^{\#}-\check{P}=-\frac{1}{\epsilon^{*}} \check{\xi}^{\#} .
$$

Substituting this expression for the logarithmic deviations of the relative target price into (19) and solving for $\xi^{\#, ~}$

$$
\check{\xi}^{\#}=-\frac{\Omega}{\omega}\left[\check{Y}-\check{Y}^{\jmath}\right],
$$

where

$$
\Omega=\frac{Y^{*} \Phi_{Y}\left(1, Y^{*}, \cdot^{*}\right)}{\Phi\left(1, Y^{*}, *^{*}\right)}
$$

and

$$
\omega=\frac{1}{\epsilon^{*}}+\frac{\mu^{\prime}(1)}{\mu(1)}+\frac{\Phi_{\xi}\left(1, Y^{*}, \cdot^{*}\right)}{\Phi\left(1, Y^{*}, \cdot^{*}\right)}
$$

Also, using (1.4) again,

$$
\check{P}^{\#}-\check{P}=\frac{\Omega}{\epsilon^{*} \omega}\left[\check{Y}-\check{Y}^{f}\right] .
$$

\subsubsection{The Instantaneously Optimal Reaction Functions}

For the sake of interpretation, it is useful to put the instantaneously optimal output and price into the language of Cooper and John (). Equation (20) implies the following instantaneously optimal reaction function for individual firm output as a function of aggregate output:

$$
\check{y}^{*}=\left[1-\frac{\Omega}{\omega}\right] \check{Y}+\frac{\Omega}{\omega} \check{Y}^{\prime} .
$$

The coefficient of $\bar{Y}$ is the slope of the reaction function, and implies a multiplier of $\frac{1}{1-\text { slope }}=\frac{\omega}{\Omega}$.

To interpret (21) as a reaction function, define $P^{f}$ as $P^{f}=M V / Y^{f}$ or

$$
\check{P}^{f}=\check{M}+\check{V}-\check{Y}^{f},
$$


so that

$$
\check{P}-\check{P}^{\prime}=-\left(\check{Y}-\check{Y}^{f}\right)
$$

Then

$$
\check{P}^{\#}=\left[1-\frac{\Omega}{\epsilon^{*} \omega}\right] \check{P}+\frac{\Omega}{\epsilon^{*} \omega} \check{Y}^{\prime},
$$

with a corresponding multiplier of $\frac{\epsilon^{*} \omega}{\Omega}$. Since the firm's price being optimal in steady state implies that $\epsilon^{*}>1$, the slope of the reaction function and the multiplier (both of which measure the degree of strategic complementarity) are greater for a price game than for an output game. The elasticities $\omega$ and $\Omega$ are the only other information needed to determine these indicators of the degree of strategic complementarity.

\subsubsection{Interpreting the Effects of Market Structure on the Target Price}

To interpret (21), think of the firm's marginal revenue and marginal cost curves. Near the steady state, $\frac{1}{\epsilon^{\bullet}}$ is the rate at which the demand curve falls, expressed as an elasticity. Since the target markup ratio $\mu$ tells how far the marginal revenue curve is below the demand curve, $\frac{1}{\epsilon^{*}}+\frac{\mu^{\prime}(1)}{\mu(1)}$ expresses the rate at which the marginal revenue curve falls, expressed as an elasticity near the steady state. On the other side, $\frac{\Phi_{\mathrm{g}}\left(1, Y^{\bullet},{ }^{\bullet}\right)}{\Phi\left(1, Y^{\bullet},{ }^{\bullet}\right)}$ is the rate at which the firm's marginal cost curve rises, expressed as an elasticity near the steady state. Thus, $\omega$ is the difference in the slopes of the marginal cost and marginal revenue curves expressed as an elasticity-or equivalently, $\omega$ is the elasticity of $\mathrm{MC} / \mathrm{MR}$ with respect to $\xi$. A $1 \%$ increase in relative output $\xi$ makes the ratio of marginal revenue to marginal cost fall by $\omega \%$.

At the macroeconomic level, $\Omega=Y^{*} \Phi_{Y} / \Phi$ is the elasticity with which a proportional increase in both aggregate output and firm output (leaving relative output $\xi$ unchanged) raises the firm's real marginal cost. On the other side, the firm's real marginal revenue is not affected at all by a proportional increase in both aggregate output and firm output that leaves relative output $\xi$ unchanged: by (16) and (14), the real price at which a firm can sell its output, and the markup that tells how much lower marginal revenue is, are both only a function of relative output $\xi$. Thus, $\Omega$ is the elasticity of $M C / M R$ with respect to a proportional increase in both aggregate output and firm output. (To be more specific, $\Omega$ is the elasticity of $\mathrm{MC} / \mathrm{MR}$ with respect to an increase in aggregate output and firm output induced by purely monetary forces in a way that leaves full employment output $Y^{f}$ unchanged.)

Now, consider a firm with $\xi=1$ that has marginal cost equal to marginal revenue at full employment output. If monetary forces cause a $1 \%$ increase in aggregate output above full employment output, with the firm's output going 
up proportionally (as it will in response to demand in the absence of any change in the firm's relative price) the average firm's marginal cost will be pushed $\Omega \%$ above its marginal revenue. Because the firm's price is fixed, there is no paradox in this gap between marginal cost and marginal revenue. However, by definition, the instantaneously optimal target output must equate marginal revenue and marginal cost. Since the elasticity of $\mathrm{MC} / \mathrm{MR}$ with respect to relative output $\xi$ is $\omega$, an $\frac{\Omega}{\omega} \%$ reduction in relative output $\xi$ would restore equality between marginal revenue and marginal cost. This $\frac{\Omega}{\omega} \%$ reduction in output to reach the target output, would be associated with an $\frac{\Omega}{c^{\circ} \omega} \%$ increase in the firm's relative price to reach the instantaneously optimal target price.

The verbal definitions of $w$ as the elasticity of MC/MR with respect to firm output, holding aggregate output fixed and $\Omega$ as the elasticity of MC/MR with respect to aggregate output when firm output moves proportionally are more general than the particular model at hand. It is useful to pay attention to these elasticities in a wide variety of models with imperfectly flexible prices.

Following Ball and Romer (1990), it is easy to relate the elasticities $\Omega$ and $\omega$ to the cost of having sticky prices. Ball and Romer's phrase "real rigidity" refers most directly to a change in aggregate output having little effect on a firm's target price, and so can be identified here with a small value of $\frac{\Omega}{c^{*} \omega}$, the elasticity of the target price with respect to aggregate output.

The triangular "dead weight loss" giving the approximate private flow cost of being away from target output has a base of length approximately $\left[\bar{\xi}_{\ell}-\bar{\xi}^{\#}\right] Y^{*}$ and a height of approximately $\left[\Omega\left(\check{Y}-\dot{Y}^{f}\right)+\omega \check{\xi}_{\ell}\right]$ marginal revenue* ${ }^{*}$. Using $(20)$, the private flow dead weight loss averaged over all firms is approximately

$$
\begin{aligned}
\frac{\mathrm{DWL}}{.5 Y^{*}\left(P^{*} / \mu^{*}\right)} \approx & \frac{\Omega^{2}}{\omega}\left(\check{Y}-\check{Y}^{\prime}\right)^{2}+\left[\Omega\left(\bar{Y}-\check{Y}^{\prime}\right)-\omega \bar{\xi}^{\#}\right] \int_{0}^{\infty} \dot{\xi}_{\ell} d \ell \\
& +\omega \int_{0}^{1}\left(\dot{\xi}_{\ell}\right)^{2} d \ell \\
= & \frac{\Omega^{2}}{\omega}\left(\check{Y}-\check{Y}^{\prime}\right)^{2}+\omega \int_{0}^{1}\left(\dot{\xi}_{\ell}\right)^{2} \cdot d \ell
\end{aligned}
$$

Of course, this private cost of being away from target output is second-orderthe only second-order quantity that I will examine in this paper. The secondterm involving the cross-sectional variance of depends on the history of the economy and so is difficult to analyze fully. However, I conjecture that at least part of the cross-sectional variance in output will be related to the variance of movements in $\xi^{\#}$, which is proportional to $\left(\frac{\Omega}{\omega}\right)^{2}$ times the variance of $\left[Y-Y^{\delta}\right]$ and so is likely to depend on $\Omega$ and $\omega$ in much the same way as the first term. ${ }^{7}$

\footnotetext{
${ }^{7}$ Steady-state inflation would introduce an additional reason for cross-sectional variance in prices and outputs. The part of the cross-sectional variation in output due to steady-state inflation should be roughly proportional to the elasticity of demand $c$ times the amount of
} 
Ball and Romer (1990) make the term involving the cross-sectional variance of output zero by using a static model in which all firms start off at the same place. In any case, a substantial part of the average private cost of sticky prices is proportional to $\frac{\Omega^{2}}{\omega}$. As Ball and Romer point out, this means that a small private cost of having sticky prices is typically associated with a small elasticity of the instantaneously optimal target price with respect to output, $\frac{\Omega}{\varepsilon^{\circ} \omega}$.

One fact that would not be apparent from reading Ball and Romer (1990) is the way in which a high initial elasticity of demand $\epsilon^{*}$ lowers the elasticity of the target price with respect to output. But in their own terms, Ball and Romer do make clear the dependence of both the private cost of having sticky prices and the elasticity of the target price with respect to output on $\Omega$ and $\omega$.

The macroeconomic elasticity $\Omega$ incorporates all of the effects of factor price pressure on marginal cost as output increases. Ball and Romer (1990) look at the possibility of increasing the macroeconomic labor supply elasticity and thereby reducing factor price pressure as output increases. In general, the elasticity $\Omega$ may also include a negative effect from production externalities and a negative effect from mechanisms that generate countercyclical target markups. In the particular model at hand, the target markup $\mu$ is acyclical, but many models exist that generate countercyclical target markups.

The microeconomic elasticity $\omega$ incorporates all aspects of increasing marginal costs and decreasing marginal revenue that are internal to the firm. Ball and Romer (1990) show that having workers strongly attached to firms ("the yeoman farmer model") leads to a lower private cost of having sticky prices than when workers are hired on the spot market. This is because when workers are strongly attached to firms, labor costs depend on firm output-a dependence that adds to $\omega$ as well as $\Omega$. If workers are always hired on the spot market, labor costs depend only on aggregate output - a dependence that adds only to $\Omega$, not $\omega$. The same principle would say that having capital strongly attached to a particular firm rather than being hired on the spot market would also reduce the private cost of having sticky prices.

The microeconomic elasticity $\omega$ also includes any dependence of the target markup on the firm's market share. Ball and Romer (1990) look at the possibility of using Woglom's (1982) model of customer markets to make the elasticity of demand fall sharply with the firm's market share (since it is harder to get new customers than to drive away old ones). This corresponds to a target markup that is sharply increasing in the firm's market share $\xi$-something that could make $\omega$ quite large. In the Neomonetarist model at hand, it is easy to find a variety aggregator $\Upsilon$ that yields a target markup that is sharply increasing in each differentiated firm's market share, reproducing the most essential implication of Woglom's (1982) model of customer markets.

inflation that would take place in the average length of time a price is fixed, all squared. This part of the cross-sectional variance of output would not be very closely related to the two parameters $\Omega$ and $\omega$. 


\section{The Short-Run Dynamics of the Neomone- tarist Model}

At a stable price steady-state, $\mathcal{P}$ and $P^{\#}$ are both equal to $P^{*}$. Therefore, dividing (2) by $P^{*}$ yields the corresponding relation in logarithmic deviations:

$$
\dot{\dot{P}}_{t}=\alpha\left[\dot{\mathcal{P}}_{t}-\check{P}_{t}\right]
$$

Similarly, with $\pi^{*}=0,(10)$ can be restated in terms of logarithmic or proportional deviations as follows:

$$
\dot{\grave{\mathcal{P}}}=\left(\alpha+r^{*}\right)\left[\check{\mathcal{P}}-\check{P}^{\#}\right] .
$$

Substituting in the logarithmic deviation of the target price given by (21), and writing $\epsilon(1)=\epsilon^{*}$ as above,

$$
\dot{\mathcal{P}}=\left(\alpha+r^{*}\right)\left[\check{\mathcal{P}}-\check{P}-\frac{\Omega}{\epsilon^{*} \omega}\left(\check{Y}-\check{Y}^{f}\right)\right] \text {. }
$$

Using the Quantity Equation to substitute in $\check{M}+\check{V}-\check{P}$ for $\check{Y}$,

$$
\dot{\overline{\mathcal{P}}}=\left(\alpha+r^{*}\right)\left[\check{\mathcal{P}}+\left(\frac{\Omega}{\epsilon^{*} \omega}-1\right) \check{P}-\frac{\Omega}{\epsilon^{*} \omega}\left(\check{M}+\check{V}-\check{Y}^{\prime}\right)\right] \text {. }
$$

As will be shown below, the fast price adjustment or high- $\alpha$ approximation I will use implies that the endogenous movements in full employment output $Y^{f}$ are slow in comparison to the price adjustment dynamics. Therefore, it is instructive to look at the short-run dynamics one obtains by treating full employment out $Y^{\prime}$ as exogenous. With $Y^{\prime}, M$ and $V$ treated as exogenous, the two endogenous variables are the optimal reset price $\mathcal{P}$ and the aggregate price level $P$ itself. Equations (22) and (25) together make a dynamic system in these two variables. It is convenient to write down the dynamics in matrix form, treating $\check{P}$ and $\check{\mathcal{P}}-\check{P}$ as the two variables:

$$
\left[\begin{array}{c}
\dot{\mathscr{P}} \\
\dot{\mathcal{P}}-\dot{P}
\end{array}\right]=\left[\begin{array}{cc}
0 & \alpha \\
\left(\alpha+r^{*}\right) \frac{\Omega}{\epsilon^{*} \omega} & r^{*}
\end{array}\right]\left[\begin{array}{c}
\check{P} \\
\check{\mathcal{P}}-\check{P}
\end{array}\right]+\left[\begin{array}{c}
0 \\
\frac{\Omega}{\epsilon^{\circ} \omega}\left(\alpha+r^{*}\right)\left(\check{Y}^{f}-\check{M}-\check{V}\right)
\end{array}\right]
$$

The absolute value of the negative eigenvalue of this matrix gives the convergence rate $\kappa$ :

$$
\begin{aligned}
\kappa & =\sqrt{\frac{\left(r^{*}\right)^{2}}{4}+\frac{\Omega}{\epsilon^{*} \omega} \alpha\left(\alpha+r^{*}\right)}-\frac{r^{*}}{2} \\
& \approx\left(\alpha+\frac{r^{*}}{2}\right) \sqrt{\frac{\Omega}{\epsilon^{*} \omega}}-\frac{r^{*}}{2}
\end{aligned}
$$


where the approximation is for $\frac{r^{*}}{\alpha}$ small. The most interesting aspect of this equation is that the convergence rate at which the economy returns to full employment after a permanent change in $M$ depends roughly on the square root of $\frac{\Omega}{\epsilon^{*} \omega}$ (the output elasticity of the target price). Thus, a high degree of "real rigidity" in Ball and Romer's (1990) sense makes price adjustment slow in comparison to the frequency $\alpha$ at which individual firms adjust their prices. If $\frac{\Omega}{\epsilon^{\circ} \omega}$ is less than 1 , aggregate price adjustment will be slower than the microeconomic rate at which firms get a chance to adjust their prices.

If $\alpha$ is varied to hold the macroeconomic rate of price adjustment $\kappa$ fixed, then a fall in $\Omega$ or an increase in $\omega$ makes price stickiness more plausible not only by reducing the private flow cost of the disequilibrium caused by a given departure of output from full employment output, but also by reducing the length of time a given firm incurs that private flow cost for each instance of fixing its price. Even an increase in $\epsilon^{*}$, for given $\Omega$ and $\omega$ would require an increase in $\alpha$ to hold $\kappa$ fixed, thus implying a shorter period of price-fixity at the firm level.

\section{The Interaction Between the Monetary Side and the Real Side of the Model}

For some purposes, the monetary side of the model are all that is needed. The real side of the Neomonetarist Model is important for three reasons. First, the real side of the model determines the elasticity $\Omega$ of marginal cost with respect to aggregate output. Second, the real side of the model shows what happens to variables other than $P$ and $Y$. Third, the real side of the model makes it possible to determine full employment output. In order to make clear what one needs to get from the real side of the model and what can be safely neglected, it is important to discuss the interaction between the monetary side of the model and the real side. For this discussion, it is sufficient to have the general outlines of the real side of the model.

\subsection{The Outlines of the Real Side}

Section 4 makes the real side of the model specific, but much that will be said in this section is more general than that specific model. Here, the only description $I$ need of the real side of the model are that the value of real marginal cost at $\xi=1$,

$$
\phi=\Phi(1, Y, \cdot)
$$

the growth rate of the capital stock $K$, and the growth rate of the marginal value of capital $\lambda$ can all be expressed as functions of $K, \lambda$ and aggregate output $Y$. Given this assumption, I can write 


$$
\check{\phi}=\varphi_{Y} \check{Y}+\varphi_{K} \check{K}+\varphi_{\lambda} \check{\lambda}
$$

The definition of full employment (18) implies that at full employment, $\phi=$ $\frac{1}{\mu(1)}=\frac{1}{\mu^{*}}$. Thus, the deviation of full employment output can be found by solving for the value of output that leaves real marginal cost $\phi$ unchanged from its steady-state value:

$$
\varphi_{Y} \check{Y}^{\prime}+\varphi_{K} \check{K}+\varphi_{\lambda} \check{\lambda}=0,
$$

which implies

$$
\check{Y}^{\prime}=-\frac{\varphi_{K}}{\varphi_{Y}} \check{K}-\frac{\varphi_{\lambda}}{\varphi_{Y}} \check{\lambda} .
$$

With full employment output $Y^{f}$ a function only of $K$ and $\lambda$, any function of $K, \lambda$ and $Y$ can be rewritten as a function of $K, \lambda$ and $Y / Y^{\prime}$. For any variable $X$, if

$$
\check{X}=x_{Y} \check{Y}+x_{K} \check{K}+x_{\lambda} \check{\lambda}
$$

then

$$
\bar{X}=x_{Y}\left(\check{Y}-\check{Y}^{j}\right)+\left[x_{K}-\frac{\varphi_{K}}{\varphi_{Y}} x_{Y}\right] \check{K}+\left[x_{\lambda}-\frac{\varphi_{\lambda}}{\varphi_{Y}} x_{Y}\right] \check{\lambda}
$$

Note that in this transformation the coefficient of $\check{Y}-\check{Y}^{f}$ is the same as the original coefficient of $\bar{Y}$. Subjecting (28) to this transformation yields the equation

$$
\check{\phi}=\varphi Y\left(\check{Y}-\check{Y}^{\prime}\right)
$$

which implies that

$$
\varphi_{Y}=\Omega
$$

Also, after making this transformation, I can write

$$
\dot{\check{K}}=\psi_{K}\left(\check{Y}-Y^{\jmath}\right)+\Psi_{K K} \check{K}+\Psi_{K \lambda} \check{\lambda}
$$

and

$$
\dot{\grave{\lambda}}=\psi_{\lambda}\left(\check{Y}-Y^{f}\right)+\Psi_{\lambda K} \check{K}+\Psi_{\lambda \lambda} \check{\lambda}
$$




\subsection{The Full Dynamic Matrix}

It is convenient to express the dynamic system in terms of the four variables $\check{P}-\check{P}^{f}, \check{P}-\check{P}, \check{K}$ and $\check{\lambda}$. The evolution of $\check{P}-\check{P}^{\prime}=-\left(\check{Y}-\check{Y}^{\prime}\right)$ is given by

$$
\begin{aligned}
\dot{P}-\dot{P}^{\prime} & =\alpha(\dot{\mathcal{P}}-\check{P})+\dot{\breve{Y}}^{\prime}-\dot{\dot{M}}-\dot{\bar{V}} \\
& =\alpha(\dot{\mathcal{P}}-\check{P})-\frac{\varphi_{K}}{\Omega} \dot{K}-\frac{\varphi_{\lambda}}{\Omega} \dot{\bar{\lambda}}-\dot{\dot{M}}-\dot{V}
\end{aligned}
$$

The full dynamic matrix is given by

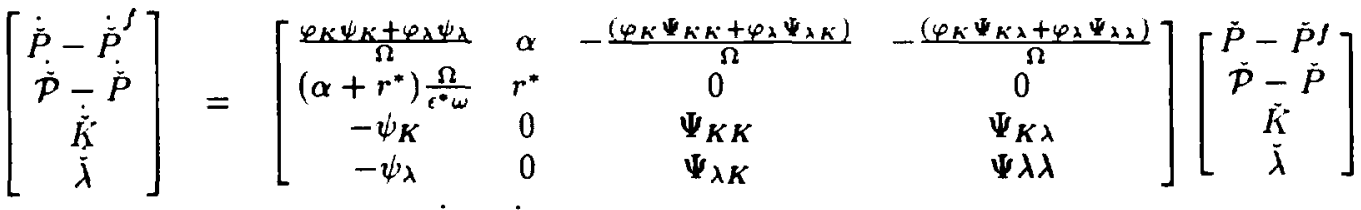

$$
\begin{aligned}
& +\left[\begin{array}{c}
-\dot{\dot{M}}-\dot{\bar{V}} \\
0 \\
0 \\
0
\end{array}\right] \text {. }
\end{aligned}
$$

\subsection{The Fast-Price-Adjustment (High- $\alpha$ ) Approximation to the Dynamic System}

To emphasize the fact that it is of order $\alpha$ as $\alpha \rightarrow \infty$, I will call the full $4 \times 4$ dynamic matrix in (33) $\alpha A$. The full dynamic matrix $\alpha A$ can be represented as a partitioned matrix of the form

$$
\begin{aligned}
\alpha A\left(\frac{1}{\alpha}\right) & =\alpha A(\iota) \\
& =\alpha\left(\left[\begin{array}{cc}
A_{11} & 0 \\
0 & 0
\end{array}\right]+\iota\left[\begin{array}{ll}
\hat{A}_{11} & \hat{A}_{12} \\
\hat{A}_{21} & \hat{A}_{22}
\end{array}\right]\right)
\end{aligned}
$$

where

$$
\begin{gathered}
\iota=\frac{1}{\alpha}, \\
A_{11}=\left[\begin{array}{cc}
0 & 1 \\
\frac{\Omega}{\epsilon^{*} \omega} & 0
\end{array}\right], \\
\hat{A}_{11}=\left[\begin{array}{cc}
\frac{\varphi \times \psi \kappa+\varphi_{\lambda} \psi_{\lambda}}{\Omega} & 0 \\
r^{*} \frac{\Omega}{\epsilon^{*} \omega} & r^{*}
\end{array}\right],
\end{gathered}
$$


and $\hat{A}_{12}, \hat{A}_{21}$ and $\hat{A}_{22}$ are the relevant $2 \times 2$ blocks of the $4 \times 4$ dynamic matrix shown in (33).

The eigenvectors of the full dynamic matrix are the key to the dynamics of the system. In general, the eigenvectors of a $4 \times 4$ matrix, while not difficult to calculate numerically, are quite hard to interpret. Thus, it is useful to make a high- $\alpha$ approximation that allows various effects to be disentangled. The principles used in making this high- $\alpha$ approximation are potentially useful in analyzing a wide variety of models in which one set of variables adjusts much faster than another set.

With $\iota=1 \alpha$, a high- $\alpha$ approximation is an expansion around $\iota=0$. The full dynamic matrix $\alpha A$ becomes infinite as $\alpha \rightarrow \infty$, but the eigenvectors of $\alpha A$ are the same as the eigenvectors of $A$, which does have a definite limit as $\alpha \rightarrow \infty$ and $\iota \rightarrow 0$. Taking $\iota$ as a parameter, the diagonal matrix of eigenvalues $\Lambda(\iota)$ and a matrix of right eigenvectors $B(\iota)$ for $A(\iota)$ must satisfy

$$
A(\iota) B(\iota)=B(\iota) \Lambda(\iota)
$$

When $\iota=0$, the upper-left corner of $A$ is $A_{11}$ and the remainder of the matrix is zero. Since the matrix is block diagonal when $\iota=0$, eigenvectors can be determined separately for the upper-left corner of the matrix and the lower-right block of the matrix. The implied separation of the dynamics of the monetary side of the model and the real side of the model that emerges as $\alpha \rightarrow \infty$ provides a formal justification for the Classical Dichotomy.

Let

$$
\Lambda(0)=\left[\begin{array}{cc}
\Lambda_{11} & 0 \\
0 & \Lambda_{22}
\end{array}\right]
$$

and

$$
B(0)=\left[\begin{array}{cc}
B_{11} & 0 \\
0 & B_{22}
\end{array}\right]
$$

Then $\Lambda_{11}$ and $B_{11}$ inust satisfy $A_{11} B_{11}=B_{11} \Lambda_{11}$. In particular, we can use the $\Lambda_{11}$ and $B_{11}$ implied by

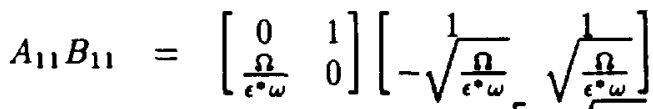

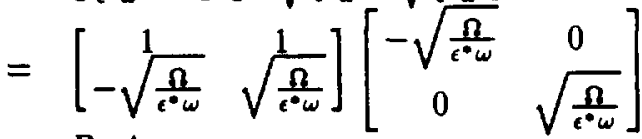

$$
\begin{aligned}
& =B_{11} \Lambda_{11}
\end{aligned}
$$

Each column of $B_{11}$ is determined only up to a scalar multiple. Generically, one can normalize the $\check{P}-\check{P}^{f}$ elements of each eigenvector to 1 , as I have done here. 
The zero block in the lower right-hand corner of $A(0)$ obviously has eigenvalues of zero, so $\Lambda_{22}$ is zero. There are many possible eigenvectors for this zero block. This enables me, conveniently, to choose a matrix of eigenvectors that are eigenvectors for the $\hat{A}_{22}$ that begins to appear in this lower-right-hand corner as $\alpha$ becomes finite and $\iota$ moves away from zero. Thus, let $\hat{\Lambda}_{22}$ be a diagonal matrix of the eigenvalues of $\hat{A}_{22}$, and let $B_{22}$ be a matrix with columns equal to two linearly independent eigenvectors of $\hat{A}_{22}$. Then $B_{22}$ satisfies $\hat{A}_{22} B_{22}=B_{22} \hat{\Lambda}_{22}$. Generically, one can normalize the $\bar{K}$ elements of $B_{22}$ to 1 .

One can see from (33) that when prices are perfectly flexible and $\check{P}-\check{P}^{\prime}$ is always zero, $\dot{K}$ and $\dot{\vec{\lambda}}$ are functions of $K$ and $\lambda$ alone and

$$
\hat{A}_{22}=\left[\begin{array}{ll}
\Psi_{K K} & \Psi_{K \lambda} \\
\Psi_{\lambda K} & \Psi \lambda \lambda
\end{array}\right]
$$

is the dynamic matrix for $K$ and $\lambda$. Thus, $\hat{\Lambda}_{22}$ and $B_{22}$ are matrices of the eigenvalues and eigenvectors for the version of the model with perfectly flexible prices.

The first-order term in the expansions of $\Lambda(\iota)$ and $B(\iota)$ are $\iota \Lambda^{\prime}(0)$ and $\iota B^{\prime}(0)$. Differentiating (34), the derivatives $\Lambda^{\prime}(0)$ and $B^{\prime}(0)$ must satisfy

$$
A(0) B^{\prime}(0)+A^{\prime}(0) B(0)=B(0) \Lambda^{\prime}(0)+B^{\prime}(0) \Lambda(0) .
$$

Using hats for the block components of $\Lambda^{\prime}(0)$ and $B^{\prime}(0)$ as for the block components of $A^{\prime}(0),(35)$ is equivalent to

$$
\begin{aligned}
{\left[\begin{array}{cc}
A_{11} & 0 \\
0 & 0
\end{array}\right] } & {\left[\begin{array}{cc}
\hat{B}_{11} & \hat{B}_{12} \\
\hat{B}_{21} & \hat{B}_{22}
\end{array}\right]+\left[\begin{array}{cc}
\hat{A}_{11} & \hat{A}_{12} \\
\hat{A}_{21} & \hat{A}_{22}
\end{array}\right]\left[\begin{array}{cc}
B_{11} & 0 \\
0 & B_{22}
\end{array}\right] } \\
= & {\left[\begin{array}{cc}
B_{11} & 0 \\
0 & B_{22}
\end{array}\right]\left[\begin{array}{cc}
\hat{\Lambda}_{11} & 0 \\
0 & \hat{\Lambda}_{22}
\end{array}\right]+\left[\begin{array}{cc}
\hat{B}_{11} & \hat{B}_{12} \\
\hat{B}_{21} & \hat{B}_{22}
\end{array}\right]\left[\begin{array}{cc}
\Lambda_{11} & 0 \\
0 & \Lambda_{22}
\end{array}\right] }
\end{aligned}
$$

or

$$
\left[\begin{array}{cc}
A_{11} \hat{B}_{11}+\hat{A}_{11} B_{11} & A_{11} \hat{B}_{12}+\hat{A}_{12} B_{22} \\
\hat{A}_{21} B_{11} & \hat{A}_{22} B_{22}
\end{array}\right]=\left[\begin{array}{cc}
B_{11} \hat{\Lambda}_{11}+\hat{B}_{11} \Lambda_{11} & 0 \\
\hat{B}_{21} \Lambda_{11} & B_{22} \hat{\Lambda}_{22}
\end{array}\right]
$$

The choice made above for $B_{22}$ as the matrix of eigenvectors of $\hat{A}_{22}$, making $\hat{\Lambda}_{22}$ the corresponding matrix of eigenvalues, already ensures that the lowerright-hand block of (36) is satisfied, regardless of the value of $\hat{B}_{22}$. Therefore, let $\hat{B}_{22}$ be zero.

The upper-right-hand block of (36) can be solved for $\hat{B}_{12}$ :

$$
\hat{B}_{12}=-A_{11}^{-1} \hat{A}_{12} B_{22} \text {. }
$$

The lower-left-hand block of (36) can be solved for $\hat{B}_{21}$ : 


$$
\hat{B}_{21}=\hat{A}_{21} B_{11} \Lambda_{11}^{-1} \text {. }
$$

The upper-left-hand block of (36) can be premultiplied by $B_{11}^{-1}$, yielding

$$
\left(B_{11}^{-1} A_{11} B_{11}\right)\left(B_{11}^{-1} \hat{B}_{11}\right)+B_{11}^{-1} \hat{A}_{11} B_{11}=\hat{\Lambda}_{11}+\left(B_{11}^{-1} \hat{B}_{11}\right) \Lambda_{11} .
$$

But since $B_{11}$ is a matrix of eigenvectors of $A_{11}$,

$$
B_{11}^{-1} A_{11} B_{11}=\Lambda_{11}
$$

and (38) can be rewritten

$$
B_{11}^{-1} \hat{A}_{11} B_{11}-\hat{\Lambda}_{11}=\left(B_{11}^{-1} \hat{B}_{11}\right) \Lambda_{11}-\Lambda_{11}\left(B_{11}^{-1} \dot{B}_{11}\right) .
$$

Distinguishing subscripted $\alpha$ 's, $\beta$ 's, carefully from the unrelated concepts denoted by plain $\alpha$ and $\beta$ to avoid confusion, define

$$
\begin{gathered}
B_{11}^{-1} \hat{A}_{11} B_{11}=\left[\begin{array}{ll}
\alpha_{11} & \alpha_{12} \\
\alpha_{21} & \alpha_{22}
\end{array}\right], \\
B_{11}^{-1} \hat{B}_{11}=\left[\begin{array}{ll}
\beta_{11} & \beta_{12} \\
\beta_{21} & \beta_{22}
\end{array}\right], \\
\Lambda(\iota)=\left[\begin{array}{cccc}
\ell_{1}(\iota) & 0 & 0 & 0 \\
0 & \ell_{2}(\iota) & 0 & 0 \\
0 & 0 & \ell_{3}(\iota) & 0 \\
0 & 0 & 0 & \ell_{4}(\iota)
\end{array}\right]
\end{gathered}
$$

Then (38) can be rewritten

$$
\left[\begin{array}{cc}
\alpha_{11}-\ell_{1}^{\prime}(0) & \alpha_{12} \\
\alpha_{21} & \alpha_{22}-\ell_{2}^{\prime}(0)
\end{array}\right]=\left[\begin{array}{cc}
0 & {\left[\ell_{2}(0)-\ell_{1}(0)\right] \beta_{12}} \\
{\left[\ell_{1}(0)-\ell_{2}(0)\right] \beta_{21}} & 0
\end{array}\right]
$$

By the diagonal, (39) implies that

$$
\begin{aligned}
\hat{\Lambda}_{11} & =\left[\begin{array}{cc}
\ell_{1}^{\prime}(0) & 0 \\
0 & \ell_{2}^{\prime}(0)
\end{array}\right] \\
& =\left[\begin{array}{cc}
\alpha_{11} & 0 \\
0 & \alpha_{22}
\end{array}\right]
\end{aligned}
$$

that is, $\hat{\Lambda}_{11}$ is equal to the main diagonal of $B_{11}^{-1} A_{11} B_{11}$. 
Since $\beta_{11}$ and $\beta_{22}$ appear nowhere in (39), they are undetermined. By choosing $\beta_{11}=-\beta_{21}$ and $\beta_{22}=-\beta_{12}$, one can normalize the $\check{P}-\check{P}^{\prime}$ elements in the top row of $B_{11}$ to zero, since the top row of $B_{11}$ is $[1,1]$ and

$$
\begin{aligned}
\hat{B}_{11} & =B_{11}\left[\begin{array}{cc}
-\beta_{21} & \beta_{12} \\
\beta_{21} & -\beta_{12}
\end{array}\right] \\
& =\frac{1}{\ell_{2}(0)-\ell_{1}(0)} B_{11}\left[\begin{array}{cc}
\alpha_{21} & \alpha_{12} \\
-\alpha_{21} & -\alpha_{12}
\end{array}\right] .
\end{aligned}
$$

\subsection{Using the Fast-Price-Adjustment Approximation to Analyze a Permanent Increase in the Money Supply}

A permanent increase in the money supply immediately pushes $P / P^{f}$ down by an equal percentage. The remainder of the dynamics take place along the saddle plane formed by the two eigenvectors with negative eigenvalues-a quickly convergent eigenvector associated with the negative eigenvalue of $\alpha A$ that is of order $\alpha$ and a slowly convergent eigenvector associated with the negative eigenvalue of $\alpha A$ that has a finite limit as $\alpha \rightarrow \infty$. In particular, the matrix

$$
B(\iota)=\left[b_{1}(\iota): b_{2}(\iota): b_{3}(\iota): b_{4}(\iota)\right] \approx\left[\begin{array}{cc}
B_{11}+\iota \hat{B}_{11} & \iota \hat{B}_{12} \\
\iota \hat{B}_{21} & B_{2} 2
\end{array}\right]
$$

can be arranged so that the first column $b_{1}(\iota)$ is the quickly convergent eigenvector and the third column $b_{3}(\iota)$ is the slowly convergent eigenvector. At time zero, the location in phase space is some linear combination of $b_{1}$ and $b_{3}$ at which $\check{P}-\check{P}^{f}$ has changed by $-\check{M}$ and $\breve{K}$ has not changed at all:

$$
\left.h_{1}(\iota) b_{1}(\iota)+h_{3}(\iota) b_{3}(\iota)=\left[\begin{array}{l}
0 \\
? \\
0 \\
?
\end{array}\right]\right]
$$

where $h_{1}$ and $h_{3}$ are scalars.

Since the $\breve{K}$ element of $b_{1}(\iota)$ is of order $\iota$, while the $\check{K}$ element of $b_{3}(\iota)$ is of order $1,(40)$ implies that the ratio $\frac{h_{1}}{h_{1}}$ is of order $\iota$. As a consequence, the ratio of the $\check{P}-\check{P}^{f}$ and $\check{\mathcal{P}}-\check{P}$ elements of $h_{3} b_{3}(\iota)$ to the $\check{P}-\check{P}^{\prime}$ element of $h_{1} b_{1}$ must be of order $\iota^{2}$. (The $\check{P}-\check{P}^{\prime}$ element of $h_{1} b_{1}$ itself must be a quantity of order 1 multiplying $\dot{M}$.) Thus, as $\alpha \rightarrow \infty$ and $\iota \rightarrow 0$, the slowly convergent eigenvector $b_{3}$ plays a negligible role in determining the deviations of $\breve{P}-\breve{P}^{f}$ and $\breve{P}-\check{P}$, either directly or by influencing the coefficient $h_{1}(\iota)$ of $b_{1}(\iota)$.

The full dynamics are given by $h_{1} b_{1} e^{\alpha t_{1} t}+h_{3} b_{3} e^{\alpha t_{3} t}$, so that the time derivative of the phase vector is 


$$
\left[\begin{array}{c}
\dot{\dot{P}}-\dot{P}^{f} \\
\dot{\bar{P}}-\dot{P} \\
\dot{\check{T}} \\
\check{\lambda}
\end{array}\right]=\alpha \ell_{1} h_{1} b_{1} e^{\alpha \ell_{1} t}+\alpha \ell_{3} h_{3} b_{3} e^{\alpha \ell_{3} t}
$$

The $\check{K}$ and $\bar{\lambda}$ elements of $h_{1} b_{1}$ and $h_{3} b_{3}$ are both of order $\iota$. But $\alpha \ell_{1}$ is of order $\alpha$ while $\ell_{3}$ is only of order 1 . Therefore, the components of $\dot{\bar{K}}$ and $\dot{\bar{\lambda}}$ arising from $\alpha \ell_{3} h_{3} b_{3}$ are only of order $\iota$; while those arising from $\alpha \ell_{1} h_{1} b_{1}$ are of order 1 -at least within the period of time of order $\iota$ that the economy is away from full employment. Thus, as $\alpha \rightarrow \infty$ and $\iota \rightarrow 0$, the slowly convergent eigenvector $b_{3}$ plays a negligible role in determining $\dot{K}$ and $\dot{\grave{\lambda}}$.

What this means is that, to a good approximation, as $\alpha \rightarrow \infty$, one can ignore the right half of the matrix $B(\iota)$ in determining the response to a permanent increase in the money supply. (Indeed, the basic principle at work here should apply to any kind of movement in the money supply or velocity that is independent of the size of $\alpha$.) Furthermore, section 3.3 demonstrates that the left-hand half of $B(\iota)$ can be determined to at least the first-order term from a knowledge of only $A_{11}, \hat{A}_{11}$ and $\hat{A}_{12}$; one does not need to know the right half of $A(\iota)$ to find the eigenvectors $b_{1}$ and $b_{2}$ to a first-order approximation. Accordingly, I can obtain a thorough picture of the effects of monetary policy as indicated by a first-order approximation of the left half of $B(\iota)$ and the left half of $A(\iota)$ while leaving the determination of the right half of $A(\iota)$ and the right half of $B(\iota)$ to another paper.

\subsection{A First-Order Approximation to Money-Driven Dy- namics}

Substituting into the formulas above,

$$
\hat{\Lambda}_{11}=\left[\begin{array}{cc}
-\frac{r^{*}}{2} \sqrt{\frac{\Omega}{\epsilon^{*} \omega}}+\frac{r^{*}}{2}+\frac{\psi_{K \varphi_{K}+\psi_{\lambda} \varphi_{\lambda}}^{2 \Omega}}{2 \Omega} & 0 \\
0 & \frac{r^{*}}{2} \sqrt{\frac{\Omega}{\epsilon^{*} \omega}}+\frac{r^{*}}{2}+\frac{\psi_{K \varphi_{K}+\psi_{\lambda} \varphi_{\lambda}} \Omega}{2 \Omega}
\end{array}\right] .
$$

Thus, taking into account endogenous changes in full employment output $Y^{f}$, but to an approximation that ignores terms in $\iota$, the quick convergence rate $\kappa$ is

$\kappa=-\alpha \ell_{1}(\iota) \approx-\alpha \ell_{1}(\iota) \approx \alpha\left[\ell_{1}(0)+\iota \ell^{\prime}(0)\right]=\left(\alpha+\frac{r^{*}}{2}\right) \sqrt{\frac{\Omega}{\epsilon^{*} \omega}}-\frac{r^{*}}{2}-\frac{\psi_{K} \varphi_{K}+\psi_{\lambda} \varphi_{\lambda}}{2 \Omega}$.

Only the last term, $-\left(\psi_{K} \varphi_{K}+\psi_{\lambda} \varphi_{\lambda}\right) /(2 \Omega)$, differs from the corresponding approximation to $\kappa$ derived above by treating $Y^{f}$ as a constant. If this term is 
positive, having output above the full employment level raises the growth rate of full employment output, making for quicker convergence, as actual output and full employment output move toward each other. The effect is cut in half because firms do not adjust prices as much when they foresee the economy being away from full employment a shorter time. ${ }^{8}$

Making the substitutions, the first column of $B(\iota)$-the quickly convergent eigenvector--is

$$
\begin{aligned}
b_{1}(\iota) \approx & {\left[\begin{array}{c}
B_{11}+\imath \hat{B}_{11} \\
\iota \hat{B}_{21}
\end{array}\right]\left[\begin{array}{l}
1 \\
0
\end{array}\right] } \\
= & {\left[\begin{array}{c}
-\left(1+\imath \frac{r^{*}}{2}\right) \sqrt{\frac{\Omega}{\epsilon^{*} \omega}}+\imath \frac{r^{*}}{2}-\imath \frac{\psi_{K} \varphi_{K}+\psi_{\lambda} \varphi_{\lambda}}{2 \Omega} \\
\iota \psi_{K} / \sqrt{\frac{\Omega}{\epsilon^{\circ} \omega}} \\
\iota \psi_{\lambda} / \sqrt{\frac{\Omega}{\epsilon^{*} \omega}}
\end{array}\right] . }
\end{aligned}
$$

It is also helpful to calculate the initial rate of change in the variables (a rate which decays thereafter at the convergence rate $\kappa$ ) in response to a permanent monetary shock. Multiplying the quickly convergent eigenvector by its eigenvalue times $-\check{M}$ (the initial disturbance in $\check{P}-\check{P}^{\prime}$ ) one finds, to an approximation that ignores terms of order $\iota$ or higher, ${ }^{9}$ that

$$
\begin{aligned}
{\left[\begin{array}{c}
\dot{P}-\dot{P}^{\prime} \\
\dot{\vec{P}}-\dot{P} \\
\dot{\check{K}} \\
\dot{\lambda}
\end{array}\right]=} & -\check{M} \alpha \ell_{1}(\iota) b_{1}(\iota) \\
\approx & {\left[\begin{array}{c}
\left(\alpha+\frac{r^{*}}{2}\right) \sqrt{\frac{\Omega}{\epsilon^{*} \omega}}-\frac{r^{*}}{2}-\frac{\psi_{K} \varphi_{K}+\psi_{\lambda} \varphi_{\lambda}}{2 \Omega} \\
r^{*} \sqrt{\frac{\Omega}{\epsilon^{*} \omega}}-\left(\alpha+r^{*}\right) \frac{\Omega}{\epsilon^{*} \omega} \\
\psi_{K} \\
\psi_{\lambda}
\end{array}\right] \check{M} }
\end{aligned}
$$

\section{The Real Side of the Neomonetarist Model}

\subsection{Investment and Capital Accumulation}

Investment adjustment costs are important to business cycle analysis in order to allow a distinction between the real interest rate and the net marginal product

\footnotetext{
${ }^{8}$ This is related to the discussion above of why the convergence rate depends approximately on the square root of $\frac{\Omega}{e w}$.

${ }^{8}$ Because the quickly convergent eigenvalue is of order $\alpha$, it was necessary to carry around terms of order $t$ until this point in order to calculate this vector to order 1.
} 
of capital. In the absence of some mechanism in a model to allow the relative price of capital and consumption goods to vary, the real interest rate and the net marginal product of capital must always be equal. Without a distinction between the real interest rate and the net marginal product of capital, virtually any stimulus to the economy, including a monetary stimulus must be associated with an increase in the real interest rate, since virtually every business cycle model implies a procyclical marginal product of capital.

I will model investment adjustment costs in the Hayashi (1982) fashion by having the rate of capital accumulation be a concave function of investment, but homogeneous in investment $I$ and the level of capital $K$ combined:

$$
\dot{K}=K J(I / K) \text {. }
$$

The rate of depreciation $\delta$ is defined as the investment to capital ratio for which $\dot{K}=0$; that is, the number satisfying

$$
J(\delta)=0
$$

Moreover, the natural way to measure the quantity of capital is so that when investment is taking place at its normal rate, here assumed to be just the replacement rate, one unit of investment creates one unit of capital on the margin. Given the normalization implicit in this way of measuring,

$$
J^{\prime}(\delta)=1 .
$$

\subsection{The Household}

Although I am pushing trend growth of the economy into the background, in order to make the model consistent with trend growth in the background, I will use the King-Plosser-Rebelo (1988) form for the utility function. An especially convenient way of expressing the King-Plosser-Rebelo utility function is

$$
\int_{0}^{\infty} e^{-\rho t} u(C, N) d t
$$

where

$$
u(C, N)=\frac{C^{\mathbf{1}-\beta}}{1-\beta} e^{(\beta-1) v(N)},
$$

or for $\beta=1$

$$
u(C, N)=\ln (C)-v(N) .
$$

This is the only type of additively time-separable utility function that allows income and substitution effects on labor supply to cancel out in the long run so 
that the trend in the real wage $W$ does not induce a trend in per capita labor $N$.

For simplicity, I will treat households as owning all of the capital and renting it out to the firms at the real rental rate $R,{ }^{10}$ and assume that each household holds an identical cross-section of the shares of the residual profits of the firms. (The households are identical in every other way, too.)

In addition to capital, a household can hold a real quantity of bonds $\Xi$ earning interest at the real rate $r$. In addition to paying for consumption $C$ and investment $I$, it must pay lump-sum taxes $T$.

Putting everything together, the representative household's problem is

$$
\max _{C, N, I} \int_{0}^{\infty} e^{-\rho t} \frac{C^{1-\beta}}{1-\beta} e^{(\beta-1) v(N)} d t,
$$

s.t.

$$
\begin{gathered}
\dot{K}=K J(I / K) \\
\Xi=r \Xi+W N+R K+I I-C-I-T,
\end{gathered}
$$

where $v^{\prime} \geq 0, v^{\prime \prime} \leq 0, J^{\prime}>0, J^{\prime \prime}<0, J(\delta)=0, J^{\prime}(\delta)=1$. If $\beta<1$, an additional restriction is needed to guarantee concavity of $u(C, N)$, but 1 will argue that the labor-constant intertemporal elasticity of substitution $s=\frac{1}{\beta}$ is less than 1 , making $\beta \geq 1$.

The current-value Hamiltonian $H$ is

$$
\begin{aligned}
H= & \frac{C^{1-\beta}}{1-\beta} e^{(\beta-1) \nu(N)}+\lambda K J\left(I / K^{*}\right) \\
& +\nu[r \Xi+W N+R K+\Pi-C-I-T],
\end{aligned}
$$

where $\lambda$ is the marginal value of a unit of capital in utils and $\nu$ is the marginal value in utils of one real dollar's worth of bonds. The marginal value of capital in dollars, $Q$, is

$$
Q=\frac{\lambda}{\nu}
$$

The first-order conditions for $C, N$ and $I$ are

$$
\begin{gathered}
C^{-\beta} e^{(\beta-1) v(N)}=\nu=\frac{\lambda}{Q} \\
v^{\prime}(N) C^{1-\beta} e^{(\beta-1) v(N)}=W \nu=W \frac{\lambda}{Q}
\end{gathered}
$$

\footnotetext{
${ }^{10}$ Whether firms or households do the investing does not matter for outcomes as long as firms would also have to buy goods for investment purposes at the retail, marked up price.
} 


$$
\lambda J^{\prime}(I / K)=\nu .
$$

The first-order conditions for $C$ and $N$ together imply

$$
W=C v^{\prime}(N),
$$

while the first-order condition for $I$ implies

$$
Q=\frac{1}{J^{\prime}(I / K)} \text {. }
$$

The Euler equation for $\lambda$ is

$$
\begin{aligned}
\dot{\lambda} & =\rho \lambda-H_{K} \\
& =\rho \lambda-\lambda J(I / K)+\lambda(I / K) J^{\prime}(I / K)-\nu R
\end{aligned}
$$

or, using (47) and (52),

$$
\frac{\dot{\lambda}}{\lambda}=\rho-J(I / K)+\frac{(I / K)-R}{Q} .
$$

The Euler equation for $\nu$ is

$$
\dot{\nu}=(\rho-r) \nu
$$

or

$$
r=\rho-\frac{\dot{\nu}}{\nu}=\rho+\frac{\dot{Q}}{Q}-\frac{\dot{\lambda}}{\lambda} .
$$

Equations (54) and (53) together guarantee that the real interest rate on bonds is equal to the rate of return on capital:

$$
r=\frac{R+\dot{Q}+Q J(I / K)-(I / K)}{Q}
$$

The expression for the return on capital in real dollars deserves some explanation. In addition to accruing capital gains and earning the rental rate, existing capital is relevant to the accumulation of further capital. By the envelope theorem, it does not matter for the calculation of this part of capital's dividend what one assumes happens to $I$ when an extra unit of capital is added. If, for convenience, one holds $I / K$ fixed, the percentage growth rate of the capital stock stays the same and an extra unit of capital adds $J(I / K)$ to the absolute accumulation of capital (valued at $Q$ per unit) but also increases the total amount (and cost) of investment by $I / K$. To obtain the rate of return, the absolute capital gains and dividends of all types need to be divided by the real price of capital $Q$. 


\subsection{The Production Function}

I will assume a generalized Cobb-Douglas production function:

$$
y_{\ell}=F\left(x_{\ell}, Y\right)=F\left(k_{\ell}^{\theta} n_{\ell}^{1-\theta}, Y\right),
$$

where $x_{\ell}$ is a Cobb-Douglas composite of the two inputs with a cost share of $\theta$ for capital and $1-\theta$ for labor as shown. The parameters $\theta$ and $1-\theta$ are the cost shares for capital and labor because for any given composite input $x_{\ell}$, the firm minimizes costs by solving

$$
\min _{k_{\ell}, n_{\ell}} W k_{\ell}+R k_{\ell}
$$

s.t.,

$$
k_{\ell}^{\theta} n_{\ell}^{1-\theta}=x_{\ell}
$$

implying among other things that

$$
\frac{W n_{\ell}}{R k_{\ell}}=\frac{1-\theta}{\theta} .
$$

In the form $n_{\ell}=\frac{R(1-\theta)}{W \theta} k_{\ell}$, this relationship can be aggregated up perfectly to the corresponding aggregate relationship:

$$
N=\frac{R(1-\theta)}{W \theta} K .
$$

The function $F$ allows the possibility of both internal and external increasing returns to scale (including fixed costs, as long as the factor composition of the fixed costs is the same as the factor composition of the variable costs). The real total cost function is of the form

$$
\frac{\mathrm{TC}}{P}=R^{\theta} W^{1-\theta} \mathcal{C}\left(y_{\ell}, Y\right)
$$

The real marginal cost $\frac{\mathrm{MC}}{P}=\Phi\left(\xi_{\ell}, Y, \cdot\right)$ at the steady-state market share $\xi_{\ell}=1$ (which determines $\Omega$ ) is therefore

$$
\phi=\Phi(1, Y, \cdot)=[R(Y, \cdot)]^{\theta}[W(Y, \cdot)]^{1-\theta} \mathcal{C}_{y}(Y, Y)
$$

The size of the effect of factor price pressure on real marginal cost will be calculated below. In addition, there is a direct effect of aggregate output on marginal cost through both arguments of $\mathcal{C}_{y}\left(y_{\ell}, Y\right)$. In the neighborhood of the steady-state, with $y_{\ell}^{*}=Y^{*}$, the elasticity $\aleph$ with which marginal cost for given factor prices falls as aggregate output increases is

$$
\kappa=-\frac{Y^{*}\left[\mathcal{C}_{y} Y\left(Y^{*}, Y^{*}\right)+\mathcal{C}_{y y}\left(Y^{*}, Y^{*}\right)\right.}{\mathcal{C}_{y}\left(Y^{*}, Y^{*}\right)}
$$


The degree of returns to scale $\gamma$ in the neighborhood of the steady-state depends only on the level of marginal cost relative to total cost, not to the derivative of marginal cost:

$$
\gamma=\frac{\mathrm{TC}}{Y^{*} \mathrm{MC}}=\frac{\mathcal{C}\left(Y^{*}, Y^{*}\right)}{Y^{*} \mathcal{C}_{y}\left(Y^{*}, Y^{*}\right)}
$$

\subsection{Steady-State Relationships Useful for Log-Linearizing Around the Steady State}

\subsubsection{The Interest Rate and Rental Rate in Steady State}

In the steady state, (45) becomes

$$
J\left(I^{*} / K^{*}\right)=0
$$

implying

$$
I^{*} / K^{*}=\delta
$$

and

$$
Q^{*}=\frac{1}{J^{\prime}\left(I^{*} / K^{*}\right)}=1
$$

Equations (53) and (54) become

$$
R^{*}=\rho+\delta
$$

and

$$
r^{*}=\rho
$$

\subsubsection{The Steady-State Composition of Output}

Let $\zeta_{C}=C^{*} / Y^{*}, \zeta_{I}=I^{*} / Y^{*}$ and $\zeta_{G}=G^{*} / Y^{*}$ be the steady state shares of consumption, investment and government purchases in output. In the steady state, the real wage and the rental rate are equal to their marginal revenue products. Since increasing returns raises the marginal product, while a markup lowers it, the steady state payments to capital as a share of output are equal to $\frac{\gamma^{*}}{\mu^{*}}$ times capital's share in costs:

$$
\frac{R^{*} K^{*}}{Y^{*}}=\theta \frac{\gamma^{*}}{\mu^{*}}
$$

(This relationship can be derived by using the fact that

$$
\mathrm{TR}=P^{*} Y^{*}=\mu^{*} \mathrm{MC} \cdot Y^{*}=\mu^{*} \frac{\mathrm{TC}}{\gamma} \text {.) }
$$


Thus,

$$
\zeta_{I}=\frac{I^{*}}{Y^{*}}=\frac{I^{*}}{K^{*}} \frac{1}{R^{*}} \frac{R^{*} K^{*}}{Y^{*}}=\frac{\delta}{\rho+\delta} \frac{\gamma}{\mu^{*}} \theta
$$

Also,

$$
\zeta_{C}=\frac{C^{*}}{Y^{*}}=\left(\frac{C^{*}}{W^{*} N^{*}}\right)\left[\frac{W^{*} N^{*}}{Y^{*}}\right]=\left(\frac{1}{\tau}\right)\left[\frac{\gamma}{\mu^{*}}(1-\theta)\right]
$$

and

$$
\frac{\zeta_{C}}{\zeta_{I}}=\frac{\rho+\delta}{\delta} \frac{1-\theta}{\theta \tau}
$$

where the notation $\tau$ for the ratio of labor income to consumption

$$
\tau=\frac{W^{*} N^{*}}{C^{*}}
$$

will be used below as well.

\subsection{Log-Linearizing Around the Steady State}

Expressing some of the key equations above in terms of logarithmic deviations from the steady state yields the following block of key equations.

For the representative household's first-order conditions, one obtains

$$
\begin{gathered}
\check{C}=s(\check{Q}-\check{\lambda})+(1-s) \tau \bar{N} \\
\check{W}=\check{C}+\frac{\check{N}}{\eta} \\
\check{Q}=j(\check{I}-\check{K})
\end{gathered}
$$

where

$$
s=\frac{1}{\beta}
$$

(as above, $s$ is the labor-constant elasticity of intertemporal substitution for consumption)

$$
\eta=\frac{v^{\prime}\left(N^{*}\right)}{N^{*} v^{\prime \prime}\left(N^{*}\right)}
$$

and

$$
j=-\frac{\left(I^{*} / K^{*}\right) J^{\prime \prime}\left(I^{*} / K^{*}\right)}{J^{\prime}\left(I^{*} / K^{*}\right)}
$$


$\eta$ is the consumption-constant elasticity of labor supply, while $j$ is the reciprocal of the elasticity of $I / K$ with respect to $Q$.

The accumulation equation for $K$ and the Euler equation for $\lambda$ yield

$$
\begin{aligned}
\dot{\dot{\lambda}} & =\frac{\dot{\tilde{\lambda}}}{\bar{\lambda}^{*}}=\widetilde{\left(\frac{\dot{\lambda}}{\bar{\lambda}}\right)} \\
& =-\left[\left(I^{*} / K^{*}\right)-R^{*}\right] \frac{\tilde{Q}}{\left(Q^{*}\right)^{2}}-\frac{\tilde{R}}{Q^{*}}+\left[\frac{1}{Q^{*}}-J^{\prime}\left(I^{*} / K^{*}\right)\right] \widetilde{\left(\frac{I}{K}\right)} \\
& =\rho \check{Q}-(\rho+\delta) \check{R}
\end{aligned}
$$

and

$$
\begin{aligned}
\dot{\dot{K}} & =\left(\overline{\frac{\dot{K}}{K}}\right) \\
& =J^{\prime}\left(I^{*} / K^{*}\right)\left[\frac{\tilde{I}}{K^{*}}-\frac{I^{*} \tilde{K}}{\left(K^{*}\right)^{2}}\right] \\
& =\left(I^{*} / K^{*}\right)[\tilde{I}-\check{K}] \\
& =\delta[\tilde{I}-\check{K}] .
\end{aligned}
$$

Moreover, (54) and (47) together imply

$$
\tilde{r}=\overline{\left(\frac{\dot{\nu}}{\nu}\right)}=-\dot{\bar{\nu}}=\dot{\bar{Q}}-\dot{\grave{\lambda}}
$$

From the production function,

$$
\check{Y}=\gamma[\theta \check{K}+(1-\theta) \check{N}]
$$

because of the constant cost shares,

$$
\check{R}=\check{W}+\check{N}-\check{K},
$$

and from (57), the logarithmic deviation of real marginal cost $\phi$ is given by

$$
\check{\phi}=\theta \check{R}+(1-\theta) \check{W}-\mathcal{N} Y .
$$

Finally, with government purchases assumed constant because there is not space here to study the effects of changes in $G, \check{G}=0$ and the closed economy accounting identity $Y=C+I+G$ can be log-linearized into

$$
\check{Y}=\zeta_{C} \check{C}+\zeta_{I} \check{I}
$$


Certain other equations are peripheral to the dynamics of the model. For example, given the material balance condition and the Ricardian equivalence implied by the model, it is not necessary to keep track of the household stock of bonds or of the government's budget constraint.

\subsection{The Short-Run Behavior of the Real Side of the Model}

Using the equations above, it is a straightforward matter to express many of the key variables in terms of $\check{N}, \check{K}$ and $\check{\nu}=\check{\lambda}-\check{Q}$. To wit,

$$
\left[\begin{array}{c}
\check{Y} \\
\check{C} \\
\check{I} \\
\check{W} \\
\check{R} \\
\check{Q}
\end{array}\right]=\left[\begin{array}{ccc}
\gamma(1-\theta) & \gamma \theta & 0 \\
\tau(1-s) & 0 & -s \\
\frac{\rho+\delta}{\delta} \frac{1-\theta}{\theta}\left(\mu^{*}-1+s\right) & \mu^{*} \frac{\rho+\delta}{\delta} & s \frac{\zeta c}{\zeta_{I}} \\
\tau(1-s)+\frac{1}{\eta} & 0 & -s \\
\tau(1-s)+\frac{1}{\eta}+1 & -1 & -s \\
j \frac{\rho+\delta}{\delta} \frac{1-\theta}{\theta}\left(\mu^{*}-1+s\right) & j\left(\mu^{*} \frac{\rho+\delta}{\delta}-1\right) & j s \frac{\zeta c}{\zeta_{I}}
\end{array}\right]\left[\begin{array}{c}
\check{N} \\
\check{K} \\
\check{\lambda}-\check{Q}
\end{array}\right]
$$

Note that

Solving the last row of $(72)$ for $\check{Q}$,

$$
\check{Q}=j \chi \frac{\rho+\delta}{\delta} \frac{1-\theta}{\theta}\left(\mu^{*}-1+s\right) \check{N}+j \chi\left(\mu^{*} \frac{\rho+\delta}{\delta}-1\right)+(1-\chi) \dot{\lambda}
$$

where

$$
\chi=\frac{1}{1+j s \frac{\zeta_{c}}{\zeta_{I}}} .
$$

Using (73), in conjunction with the fact that

$$
\check{N}=\frac{\check{Y}}{\gamma(1-\theta)}-\frac{\theta}{1-\theta} \check{K}
$$

one can express the behavior of the model in terms of $\check{Y}, \check{K}$ and $\check{\lambda}$ as follows: 


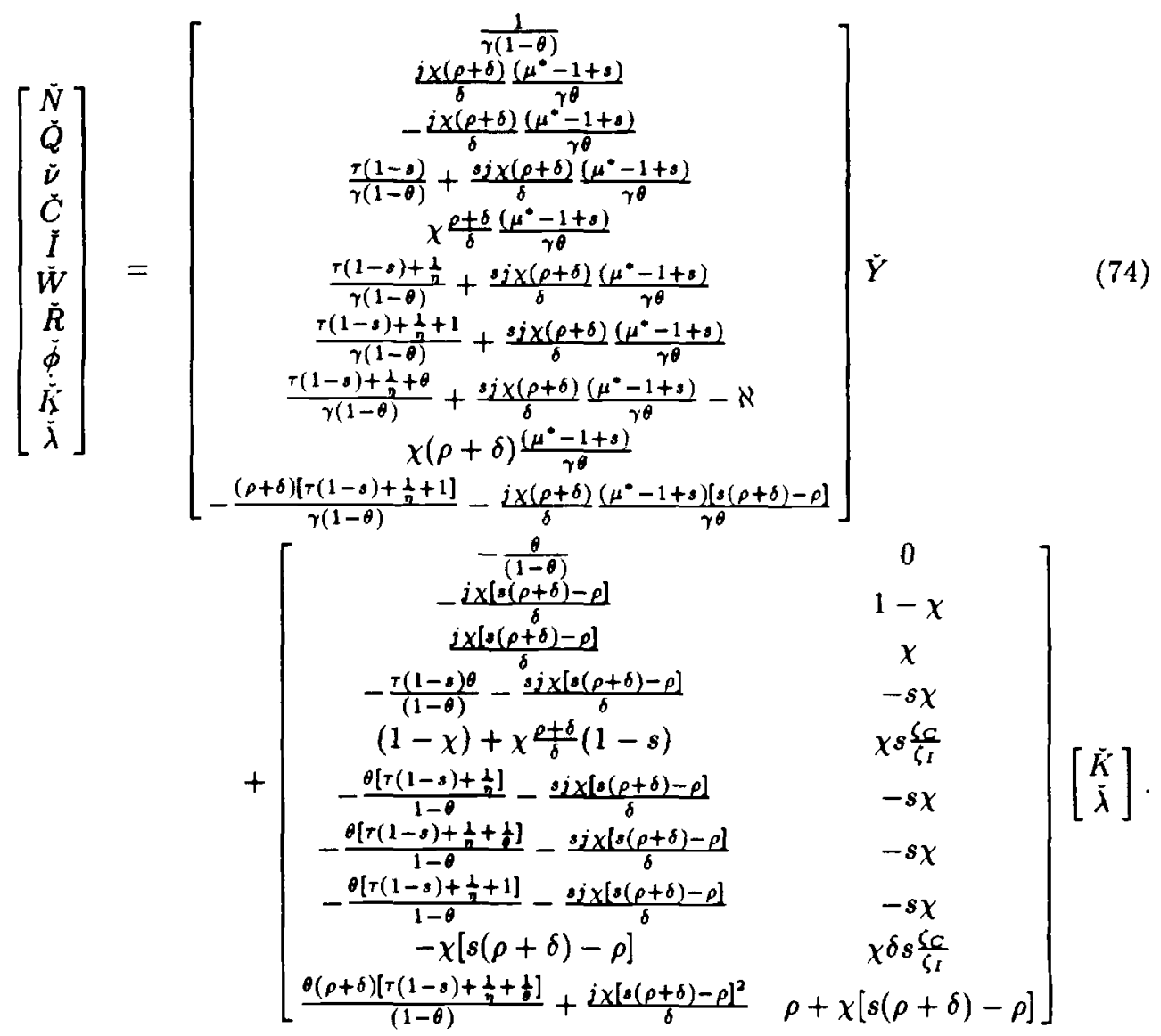

From (74)

$$
\Omega=\varphi_{Y}=\frac{\tau(1-s)+\frac{1}{\eta}+\theta}{\gamma(1-\theta)}+\frac{s j \chi(\rho+\delta)}{\delta} \frac{\left(\mu^{*}-1+s\right)}{\gamma \theta}-\aleph .
$$

Of the three major terms in $\Omega$, the first stems from the upward slope of the labor supply curve (in part due to the consumption-constant labor supply elasticity being less than infinity and in part due to consumption rising with output) plus the fact that the rental rate of capital rises even faster with output than the shadow real wage. The second major term stems from investment adjustment costs. The third major term, $-\mathcal{N}$, stems from any tendency of marginal cost to decline with aggregate output for constant factor prices. Unless $N$ is quite a large negative number (marginal cost falling with aggregate output) -or in a more general model, some mechanism leading to countercyclical target markups 
is quite large-the elasticity $\Omega$ is likely to be greater than 1 . Therefore, in the model at hand, the easiest way to make $\frac{\Omega}{\epsilon^{*} \omega}$ small is to make $\epsilon^{*} \omega$ large. Making $\epsilon^{*} \omega$ large while $\Omega$ remains a substantial positive number raises the degree of strategic complementarity and real rigidity without leading to the multiple equilibria that typically arise when $\Omega$ is negative.

A positive value of $\Omega$ means that real marginal costs are positively related to the excess of output over full employment output. Assuming that $s \leq 1$, (74) implies that all of the other variables in the vector on the left-hand side are positively related to excess output except $\nu$, which is negatively related to excess output, and $\dot{\dot{\lambda}}$, which is ambiguously related to excess output. (If $s \leq 1$, $\dot{\bar{\lambda}}$ is negatively related to excess output unless $s$ is quite small and $j$ is quite large.)

Finally, (74) implies that

$$
\begin{aligned}
\varphi_{K} & =-\frac{\theta\left[\tau(1-s)+\frac{1}{\eta}+1\right]}{1-\theta}-\frac{s j \chi[s(\rho+\delta)-\rho]}{\delta} \\
\varphi_{\lambda} & =-s \chi, \\
\psi_{K} & =\chi(\rho+\delta) \frac{\left(\mu^{*}-1+s\right)}{\gamma \theta} \\
\psi_{\lambda} & =-\frac{(\rho+\delta)\left[\tau(1-s)+\frac{1}{\eta}+1\right]}{\gamma(1-\theta)}-\frac{j \chi(\rho+\delta)}{\delta} \frac{\left(\mu^{*}-1+s\right)[s(\rho+\delta)-\rho]}{\gamma \theta}
\end{aligned}
$$

\subsection{The Determination of the Real Interest Rate}

The easiest way to determine the behavior of the real interest rate is from (67):

$$
\tilde{r}=-\dot{\bar{\nu}} \text {. }
$$

From (74),

$$
\check{\nu}=v_{Y} \check{Y}+v_{K} \check{K}+v_{\lambda} \check{\lambda}
$$

where

$$
\begin{gathered}
v_{Y}=-\frac{j \chi(\rho+\delta)}{\delta} \frac{\left(\mu^{*}-1+s\right)}{\gamma \theta} \\
v_{K}=\frac{j \chi[s(\rho+\delta)-\rho]}{\delta}
\end{gathered}
$$

and

$$
v_{\lambda}=\chi=\frac{1}{1+j s \frac{\zeta_{c}}{\zeta_{I}}} .
$$


Based on the transformation implicit in (29) and the approximate derivative of quickly convergent eigenvector given in (42) (plus (30) and (58)),

$$
\begin{aligned}
\tilde{r} & =-v_{Y} \dot{\dot{Y}}-v_{K} \dot{\dot{K}}-v_{\lambda} \dot{\dot{\lambda}} \\
& =-v_{Y}\left[\dot{\bar{Y}}-\dot{\bar{Y}} \jmath+\left[\frac{\varphi_{K}}{\Omega} v_{Y}-v_{K}\right] \dot{K}+\left[\frac{\varphi_{\lambda}}{\Omega} v_{Y}-v_{\lambda}\right] \dot{\bar{\lambda}}\right. \\
& \approx\left\{v_{Y}\left[\left(\alpha+\frac{\rho}{2}\right) \sqrt{\frac{\Omega}{\epsilon^{*} \omega}}-\frac{\rho}{2}+\frac{\psi_{K} \varphi_{K}+\psi_{\lambda} \varphi_{\lambda}}{2 \Omega}\right]-v_{K} \psi_{K}-v_{\lambda} \psi_{\lambda}\right\} \dot{M}
\end{aligned}
$$

The coefficient of $\alpha$ is $v_{Y} \sqrt{\frac{\Omega}{c^{-} \omega}}$ which is strictly negative if $j>0$. Thus, as long as there is some investment adjustment cost, a high enough value of $\alpha$ can guarantee that a monetary expansion will cause the real interest rate to fall. Below, I investigate just how fast price adjustment must be for this to happen.

\section{Calibration}

\subsection{Parameter Values}

Because the model has been solved algebraically, it is easy to plug in different sets of parameter values. Based on microeconomic evidence, I have a serious disagreement with two of the parameter values traditional in the Real Business Cycle literature-the traditional values for the elasticity of intertemporal substitution and for the labor supply elasticity. Also, by default, the investment adjustment cost parameter is typically set to zero. Having introduced investment adjustment costs, I can follow the microeconomic evidence suggesting a non-zero value for $j$. These parameters I discuss further below.

Based on Basu (1993), Basu and Fernald (1994a,b) and Basu and Kimball (1995), I choose $\gamma=1.1$ and $\mu^{*}=1.1$, implying $\epsilon^{*}=11$. (With $\gamma / \mu^{*}=1$ the steady state has zero pure profits.) In the absence of strong evidence against constant marginal cost, and the absence of any strong evidence of productive externalities, I choose $\aleph=0$. Thus, in these parameters, I assume only a modest departure from constant returns to scale and perfect competition.

The other, less controversial parameter values are assigned as follows: $\theta=$ $\left(\gamma / \mu^{*}\right)(\rho+\delta) K^{*} / Y^{*}=.3,1-\theta=\left(\gamma / \mu^{*}\right) W^{*} N^{*} / Y^{*}=.7, \delta=.08 /$ year, $\rho=$ $.02 /$ year, and $\tau=W^{*} N^{*} / C^{*}=1$. These imply $\zeta_{C}=C^{*} / Y^{*}=(1-\theta) / \tau=.7$, $\zeta_{I}=I^{*} / Y^{*}=\delta \theta /(\rho+\delta)=.24$ and $\zeta_{G}=G^{*} / Y^{*}=1-\zeta_{C}-\zeta_{I}=.06$. (The value .06 is too low for the share of all government purchases in output, but seems reasonable for the share of government purchases that are not good substitutes for private consumption and investment.)

The two parameters most difficult to calibrate are the microeconomic rate of price adjustment $\alpha$ and the ratio $\mu^{\prime}(1) / \mu(1)$, which is a key component of $\omega$. (Given the assumption of constant marginal costs, making $\Phi_{\xi}=0$, 


$$
\left.\omega=\frac{1}{\epsilon^{*}}+\frac{\mu^{\prime}(1)}{\mu(1)}=.09+\frac{\mu^{\prime}(1)}{\mu(1)} .\right)
$$

Therefore, $\alpha$ and $\omega$ will be left as algebraic symbols for now. It is useful, however, to remember that in order to make sticky prices plausible, large values of $\omega$ are needed. To get some sense of the possible values of $\omega$, note that a value of 10 for $\mu^{\prime}(1) / \mu(1)$, making $\omega=10.09$, would require a $1 \%$ increase in market share $\xi$ to cause a fall in the elasticity of demand from 11 to 6 , so that $\mu$ would increase from 1.1 to 1.2 .

\subsubsection{The Elasticity of Intertemporal Substitution and Additive Non- separability between Consumption and Labor}

A large percentage of Real Business Cycle models assume an elasticity of intertemporal substitution $s$ equal to 1, which is implausibly high. ${ }^{11}$ Empirically, no one has been able to establish conclusively that the real interest rate has any effect on the timing of consumption, much less that a one percent increase in the real interest rate increases the expected growth rate of consumption by anything close to the one percent figure predicted if the elasticity of intertemporal substitution were equal to one. Given the standard errors in, say, Hall's (1988) regressions, the failure to find intertemporal substitution empirically is quite consistent with some positive elasticity of intertemporal substitution as one must insist on on theoretical grounds, but it is not consistent with an elasticity of intertemporal substitution equal to one. Even if the standard model is failing to some extent, an elasticity of intertemporal substitution as large as one should be enough to cut through a lot of model noise. Responses to survey questions based on choices in hypothetical situations confirm these econometric results (see Barsky, Juster, Kimball and Shapiro, 1995).

The primary justification for assuming an elasticity of intertemporal substitution equal to one is that this is the only value for which the King-PlosserRebelo (1988) utility functions yields additive separability between consumption and labor. But I see no compelling reason to assume additive separability between consumption and labor. Indeed, when tested, additive separability is often rejected (See for example the mimeo by Garcia, Lusardi and $\mathrm{Ng}, 1994$.) Within the class of King-Plosser-Rebelo preferences, an elasticity of intertemporal substitution less than one implies that for any given level of consumption laboring more intensely raises the marginal utility of consumption. To me, this seems quite plausible. In a permanent income setting, it means that additional labor induced by a temporary increase in the shadow real wage tends to induce additional consumption. The positive effect of labor on consumption implied by a King-Plosser-Rebelo utility function with elasticity of substitution less than

\footnotetext{
"Some papers, notably King, Plosser and Rebelo (1988) depart from this default value of one, but the traditional value of one continually reasserts itself.
} 
one gives it a fighting chance at explaining the business cycle fact that consumption and labor tend to move in the same direction. As one turns to a model with a smaller elasticity of intertemporal substitution, interest rate effects become less important as demanded by the empirical evidence and effects induced by fluctuations in the shadow real wage become even more important.

Therefore, to be consistent with Hall (1988) and Barsky, Juster, Kimball and Shapiro (1995), let $s=.2$.

\subsubsection{The Consumption-Constant Elasticity of Labor Supply}

Typical microeconomic estimates of the labor supply elasticity are quite low. However, the regressions behind these estimates either use observed high-frequency variations in observed wages, confounding the observed real wage with the shadow real wage, or they rely on life-cycle variations in the real wage. The effects of life-cycle variations in the real wage are difficult to disentangle from other age-linked effects.

In the absence of clear microeconomic evidence, the Real Business Cycle literature has traditionally chosen quite high values of the labor supply elasticity, because those large values make it easier to fit the macroeconomic data. There is too little space here to present the critique by Hall (1987) and others of the Rogerson (1984) justification for high macroeconomic labor supply elasticities. Instead, let me suggest a disciplined way of judging the appropriate size of the consumption-constant labor supply elasticity which is robust to the presence of Rogersonian nonconvexities at the microeconomic level.

Whatever its microeconomic underpinnings, to be consistent with steadystate growth, the macroeconomic labor supply relation should imply that the income and substitution effects of permanently higher real wages cancel. This allows one to guage the size of the substitution effect by thinking about the size of the income effect.

If there is still a representative household, it should have a King-PlosserRebelo (1988) utility function, as above. The slope of the income expansion path can be identified by looking at how consumption and labor are related when the (shadow) real wage is held constant. By (63), when $\check{W}=0$,

$$
\check{N}=-\eta \check{C} .
$$

The as income increases, the additional expenditure on consumption is

$$
\tilde{C}=C^{*} \check{C}
$$

while the additional expenditure on leisure is

$$
-W^{*} \tilde{N}=-C^{*}\left(\frac{W^{*} N^{*}}{C^{*}}\right) \check{N}=-C^{*} \tau \bar{N}=C^{*} \tau \eta \check{C} .
$$

Thus, the marginal expenditure share of consumption $h$ is 


$$
h=\frac{\tilde{C}}{\tilde{C}-W^{*} \tilde{N}}=\frac{1}{1+\tau \eta}
$$

and the marginal expenditure share of leisure is

$$
1-h=\frac{\tau \eta}{1+\tau \eta}
$$

Given either of these marginal expenditure shares, the consumption-constant labor supply elasticity can be calculated as

$$
\eta=\frac{1-h}{\tau h}
$$

With $\tau=\frac{W^{*} N^{*}}{C^{*}}=1$, the labor supply elasticity $\eta$ is equal to the ratio of the marginal expenditure share of leisure $1-h$ to the marginal expenditure share of consumption $h$.

The thought experiment to get these marginal expenditure shares is to think how one would spend the proceeds of lottery winnings. What percentage would be spent on increased consumption and what percentage would be spent on reduce work hours. If income and substitution effects are to cancel, anyone who wishes to argue for a high labor supply elasticity must also be prepared to defend the idea that people would spend a substantial fraction of such windfalls on reduced work. For what it is worth, a sample of students asked to imagine a future situation in which they were working had an average marginal expenditure share of leisure equal to about .25 . Even though the modal response was zero, many indicated they would spend a substantial fraction of a windfall on reducing work hours. This implies a consumption-constant labor supply elasticity of about $\frac{25}{75}=.33$. A marginal expenditure share of leisure of .5 , which is as high as seems at all plausible to me, would yield a consumption-constant labor supply elasticity of about 1 . The only way to get the infinite labor supply elasticity that appears in some Real Business Cycle Models and still have income and substitution effects cancel is to have a marginal expenditure share of leisure equal to 1 . That is, those models imply that a household would spend $100 \%$ of a windfall on reducing labor supply. Or, to the extent that the high labor supply elasticity arises from mechanisms at a higher level than that of the individual household, it is true at least that $100 \%$ of a windfall to the class of workers as a whole would be spent on reducing labor. This is a typically unnoticed feature of these models. ${ }^{12}$ In order to get the highest plausible value of $\eta$, take the extreme case in which half of a windfall is spent on consumption and half on leisure $(h=.5)$, so that $\eta=1$.

\footnotetext{
${ }^{12}$ As a note on the history of thought, this method of calibrating the labor supply elasticity from the marginal expenditure share of leisure is closely akin to the way in which Prescott (1986) calibrates the labor supply elasticity. However, Prescott (1986) calibrates the labor supply elasticity using the average expenditure shares of leisure and consumption, with the implicit assumption that the average and marginal expenditure shares are equal. This gave
} 


\subsubsection{Investment Adjustment Costs}

By (64), the elasticity $I / K$ with respect to $Q$ is $\frac{1}{j}$. Regressions of $I / K$ on $Q$ have often found quite low coefficients, implying quite high values of $j$. However, these high values of $j$ give an implausibly slow partial equilibrium rate of convergence of the capital stock to the target capital stock.

Consider a simple partial equilibrium model of cost-minimization, in which a firm's output is taken as exogenous, the interest rate $r$ is exogenous and constant, and the marginal revenue product $R$ is given by

$$
\check{R}=(\check{y}-\check{k})+b \check{y} .
$$

The production function is basically Cobb-Douglas, but the marginal revenue product can also be affected by the scale at which the firm operates. The rate of change of the firm's capital stock is given by

$$
\dot{\tilde{k}}=\frac{\delta}{j} \check{Q}
$$

since $\frac{Q}{j}$ is the logarithmic deviation of the investment to capital ratio and $\delta$ is the base rate for the investment to capital ratio. Tobin's $Q$ is given by

$$
\bar{Q}_{t}=R^{*} \int_{t}^{\infty} e^{-r(\sigma-t)}\left[(1+b) \check{y}_{\sigma} \check{k}_{\sigma}\right] d \sigma .
$$

Using Leibniz' rule,

$$
\dot{Q}=R^{*}[\check{Q}+\check{k}-(1+b) \check{y}]
$$

In matrix form,

$$
\left[\begin{array}{l}
\dot{\bar{k}} \\
\check{Q}
\end{array}\right]=\left[\begin{array}{ll}
0 & \delta \\
r & \dot{j}
\end{array}\right]\left[\begin{array}{l}
\check{k} \\
\dot{Q}
\end{array}\right]+\left[\begin{array}{c}
0 \\
-(1+b) r \grave{y}
\end{array}\right] \text {. }
$$

The absolute value of the negative eigenvalue for this partial equilibrium model is

$$
\sqrt{\frac{r^{2}}{4}+R^{*} \frac{\delta}{j}}-\frac{r^{*}}{2}
$$

. If $j$ were as large as 1 , then (with $r^{*}=\rho=.02 /$ year, $\delta=.08 /$ year and $R^{*}=$ $\rho+\delta=.10 /$ year) the partial equilibrium adjustment rate would be $.0905 /$ year,

rise to the question of how to deal with sleep in calculating the average expenditure share of leisure, something that makes an enormous difference in the implied labor supply elasticity. But the question of how to deal with sleep can be seen for the red herring it is once one focuses appropriate attention on the marginal expenditure shares as opposed to the average expenditure shares. 
making the adjustment half-life to the target capital stock more than sevenand-a-half years.

Using a new method of identification, Cummins, Hassett and Hubbard (1994) find much larger elasticities of $I / K$ with respect to $Q-$ on the order of 5 , which would imply $j=.2$, and an adjustment half-life of 3.24 years. With capital being a mixture of equipment and structures, this seems within the bounds of reason. Therefore, let $j=.2$.

\subsection{General Equilibrium Elasticities Implied by these $\mathbf{P a}$ - rameter Values}

Substituting in the chosen parameter values, the short-run behavior of the model is given by

$$
\left[\begin{array}{c}
\tilde{N} \\
\check{Q} \\
\check{\nu} \\
\check{C} \\
\check{I} \\
\breve{W} \\
\check{R} \\
\check{\phi} \\
\check{K} \\
\check{\lambda}
\end{array}\right]=\left[\begin{array}{ccc}
1.277 & -.429 & 0 \\
.203 & 0 & .105 \\
-.203 & 0 & .895 \\
1.08 & -.343 & -.179 \\
1.017 & 1 & .522 \\
2.379 & -.771 & -.179 \\
3.677 & -2.2 & -.179 \\
2.768 & -1.2 & -.179 \\
.0814 & 0 & .042 \\
-.364 & .22 & .02
\end{array}\right]\left[\begin{array}{c}
\check{Y} \\
\check{K} \\
\check{\lambda}
\end{array}\right]
$$

The full dynamic matrix is

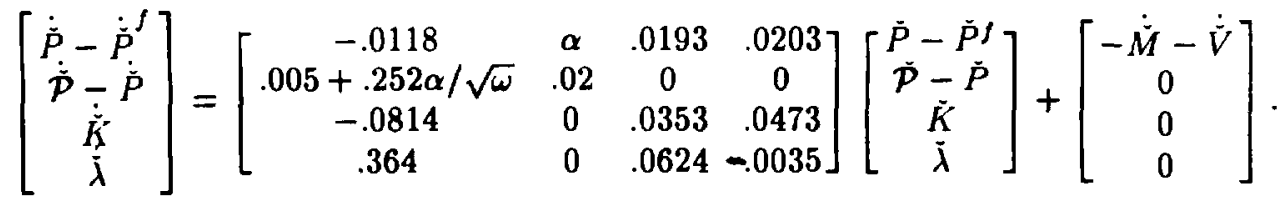

\subsection{Does the Real Interest Rate Fall in Response to a Monetary Stimulus?}

Using the high- $\alpha$ approximation developed above, the absolute value of the quickly convergent eigenvalue is given by

$$
\kappa \approx .5016 \frac{(\alpha+.01)}{\sqrt{\omega}}-.0041,
$$

and the effect of a permanent increase in the money supply on the real interest rate is given by 


$$
\tilde{r} \approx\left[.329-.0322 \frac{\alpha+.01}{\sqrt{\omega}}\right] \check{M}
$$

or

$$
\tilde{r} \approx[.3289-.0642 \kappa] \check{M} .
$$

Thus, the aggregate price level would have to converge at a rate of at least $.3289 / .0642=5.123$, or more than $500 \%$ per year in order for a permanent increase in $M$ to cause a fall in the real interest rate. In other words, unless the half-life for macroeconomic price-adjustment is less than $\mathbf{5 0}$ days, a monetary expansion should lead to an increase in the real interest rate. In terms of intermediate macroeconomics, the IS curve is stubbornly upward-sloping. It may be necessary to model any real-world tendency for the real interest rate to fall in response to a monetary stimulus as a result of output being temporarily off the IS curve.

\section{Conclusion}

Though there is much more that could be said about the Neomonetarist model above, several things are apparent.

First, an elasticity of demand falling sharply with a firm's market share is both easy to model and effective at reducing the private cost of price stickiness, making price stickiness more plausible.

Second, mechanisms that increase real rigidity and thereby make sticky prices more plausible also tend to slow down the general equilibrium adjustment of prices. This general equilibrium adjustment rate is not the same as the rate $\alpha$ at which individual firms are assumed to change their prices. If there is enough real rigidity to make sticky prices plausible, the general equilibrium adjustment rate is likely to be significantly slower than $\alpha$.

Third, even when investment adjustment costs are introduced to give the real interest rate a fighting chance to fall in response to a monetary expansion, plausible parameter values imply that the real interest rate will increase in response to a monetary expansion. 


\section{References}

Ball, Laurence, and David Romer. "Real Rigidities and the Non-Neutrality of Money." Review of Economic Studies, 57 (April 1990), 183-203.

Barsky, Robert, F. Thomas Juster, Miles S. Kimball and Matthew Shapiro. "An Experimental Approach to Preference Parameters and Behavioral Heterogeneity in the Health and Retirement Study." Mimeo, University of Michigan, 1995.

Basu, Susanto. “Cyclical Productivity: Overhead Inputs or Cyclical Utilization?" Mimeo, University of Michigan, 1993.

Basu, Susanto, and John Fernald. "Are Apparent Productive Spillovers a Figment of Specification Error?" International Finance Discussion Paper 463, Federal Reserve Board, 1994a.

Basu, Susanto and John Fernald. "Constant Returns and Small Markups in U.S. Manufacturing." International Finance Discussion Paper 463, Federal Reserve Board, 1994b.

Basu, Susanto, and Miles S. Kimball. "Cyclical Productivity with Unobserved Input Variation." Mimeo, University of Michigan, 1995.

Calvo, Guillermo. "On the Microfoundations of Staggered Nominal Contracts: A First Approximation." Mimeo, Columbia University, January 1982.

Cummins, Jason G., Kevin A Hassett, and R. Glenn Hubbard. "A Reconsideration of Investment Behavior Using Tax Reforms as Natural Experiments." Mimeo, Columbia University, April 1994.

Friedman, Milton, and Anna J. Schwartz. A Monetary History of the United States, 1867-1960, Princeton: Princeton University Press, 1963.

Garcia, René, Annamaria Lusardi, and Serena Ng. "Excess Sensitivity and Asymmetries in Consumption: An Empirical Investigation." Mimeo, University of Montreal, June 1994.

Hall, Robert. "The Volatility of Employment with Fixed Costs of Going to Work." Mimeo, Stanford, June 1987.

Hall, Robert. "Intertemporal Substitution in Consumption." Journal of Political Economy, 96 (April 1988), 339-357.

Hayashi, Fumio. "Tobin's Marginal and Average q: A Neoclassical Interpretation." Econometrica, 50 (January 1982), 213-224.

Kimball, Miles S., and Philippe Weil. Macroeconomics and Finance: A Dynamic and Stochastic Optimization Approach. Forthcoming, Cambridge: MIT Press. 
King, Robert G., Charles I. Plosser and Sergio T. Rebelo. "Production, Growth and Business Cycles: I. The Basic Neoclassical Model." Journal of Monetary Economics 21 (March 1988), 195-232.

Prescott, Edward. "Theory Ahead of Business Cycle Measurement." Federal Reserve Bank of Minneapolis Quarterly Review, 10 (Fall 1986), 9-22.

Rogerson, Richard. "Indivisible Labor, Lotteries, and Equilibrium." Chapter 1 of Topics in the Theory of Labor Markets, Ph.D. dissertation, University of Minnesota, 1984.

Weitzman, Martin L. The Share Economy, Cambridge, Harvard University Press, 1984.

Woglom, Geoffrey. "Underemployment Equilibrium with Rational Expectations." Quarterly Journal of Economics, 97 (1982), 89-107. 